\title{
Hybrid Scheduling for Multi-Equipment at U-Shape Trafficked Automated Terminal Based on Chaos Particle Swarm Optimization
}

\author{
Junjun Li ${ }^{1, *}$, Jingyu Yang ${ }^{2}$, Bowei Xu ${ }^{2} \mathbb{D}$, Yongsheng Yang ${ }^{2}$, Furong Wen ${ }^{3}$ and Haitao Song ${ }^{3}$ \\ 1 Merchant Marine College, Shanghai Maritime University, Shanghai 201306, China \\ 2 Institute of Logistics Science and Engineering, Shanghai Maritime University, Shanghai 201306, China; \\ 201930510021@stu.shmtu.edu.cn (J.Y.); bwxu@shmtu.edu.cn (B.X.); yangys@shmtu.edu.cn (Y.Y.) \\ 3 Technical Department, Beibu Gulf Port Co., Ltd., Nanning 530000, China; wenfr@bbwport.com (F.W.); \\ songht@bbwport.com (H.S.) \\ * Correspondence: 1ijj@shmtu.edu.cn
}

\section{check for} updates

Citation: Li, J.; Yang, J.; Xu, B.; Yang, Y.; Wen, F.; Song, H. Hybrid Scheduling for Multi-Equipment at U-Shape Trafficked Automated Terminal Based on Chaos Particle Swarm Optimization. J. Mar. Sci. Eng. 2021, 9, 1080. https://doi.org/ 10.3390/jmse9101080

Academic Editors: Fausto Pedro García Márquez, Mayorkinos Papaelias and Simone Marini

Received: 7 September 2021

Accepted: 28 September 2021

Published: 1 October 2021

Publisher's Note: MDPI stays neutral with regard to jurisdictional claims in published maps and institutional affiliations.

Copyright: (c) 2021 by the authors. Licensee MDPI, Basel, Switzerland. This article is an open access article distributed under the terms and conditions of the Creative Commons Attribution (CC BY) license (https:/ / creativecommons.org/licenses/by/ $4.0 /)$.
Abstract: Aimed to improve the efficiency of port operations, Shanghai Zhenhua Heavy Industries Co., Ltd. (ZPMC) proposed a new U-shape trafficked automated terminal. The new U-shape trafficked automated terminal brings a new hybrid scheduling problem. A hybrid scheduling model for yard crane (YC), AGV and ET in the U-shape trafficked automated terminal yard is established to solve the problem. The AGV and ET yard lanes are assumed to be one-way lane. Take the YC, AGV and ET scheduling results (the container transportation sequences) as variables and the minimization of the maximum completion time as the objective function. A scheduling model architecture with hierarchical abstraction of scheduling objects is proposed to refine the problem. The total completion time is solved based on a static and dynamic mixed scheduling strategy. A chaotic particle swarm optimization algorithm with speed control (CCPSO) is proposed, which include a chaotic particle strategy, a particle iterative speed control strategy, and a particle mapping space for hybrid scheduling. The presented model and algorithm were applied to experiments with different numbers of containers and AGVs. The parameters of simulation part refer to Qinzhou Port. The simulation results show that CCPSO can obtain a near-optimal solution in a shorter time and find a better solution when the solution time is sufficient, comparing with the traditional particle swarm optimization algorithm, the adaptive particle swarm optimization algorithm and the random position particle swarm optimization algorithm.

Keywords: U-shape trafficked container terminal; static and dynamic mixed scheduling; particle swarm optimization; chaos mapping

\section{Introduction}

In the booming global logistics, the container terminals, as important parts of ports, are connected to both land and sea transportation. In the increasingly competitive environment, the requirements for container terminal operations are also getting higher and higher. As shown in Figure 1, in the traditional terminal, the yard's handover areas are set at the ends of each yard block. External trucks (ETs) and automated guided vehicles' (AGVs) handover are on the land side and the sea side, respectively, and they cannot enter into the yard. The container is transported from/to the AGV or ET to/from the yard crane at the yard block end. The following situations can easily to happen: (1) YCs are always in the state of carrying containers with heavy loads over long distances; (2) YCs are easy to interfere with each other, and it is difficult to take care of loading and unloading ships; (3) the roads of AGV and ET are not completely separated, which is easy to cause congestion; and (4) when ET enters the handover area of the yard, it needs to reverse parking, which is difficult to operate. 


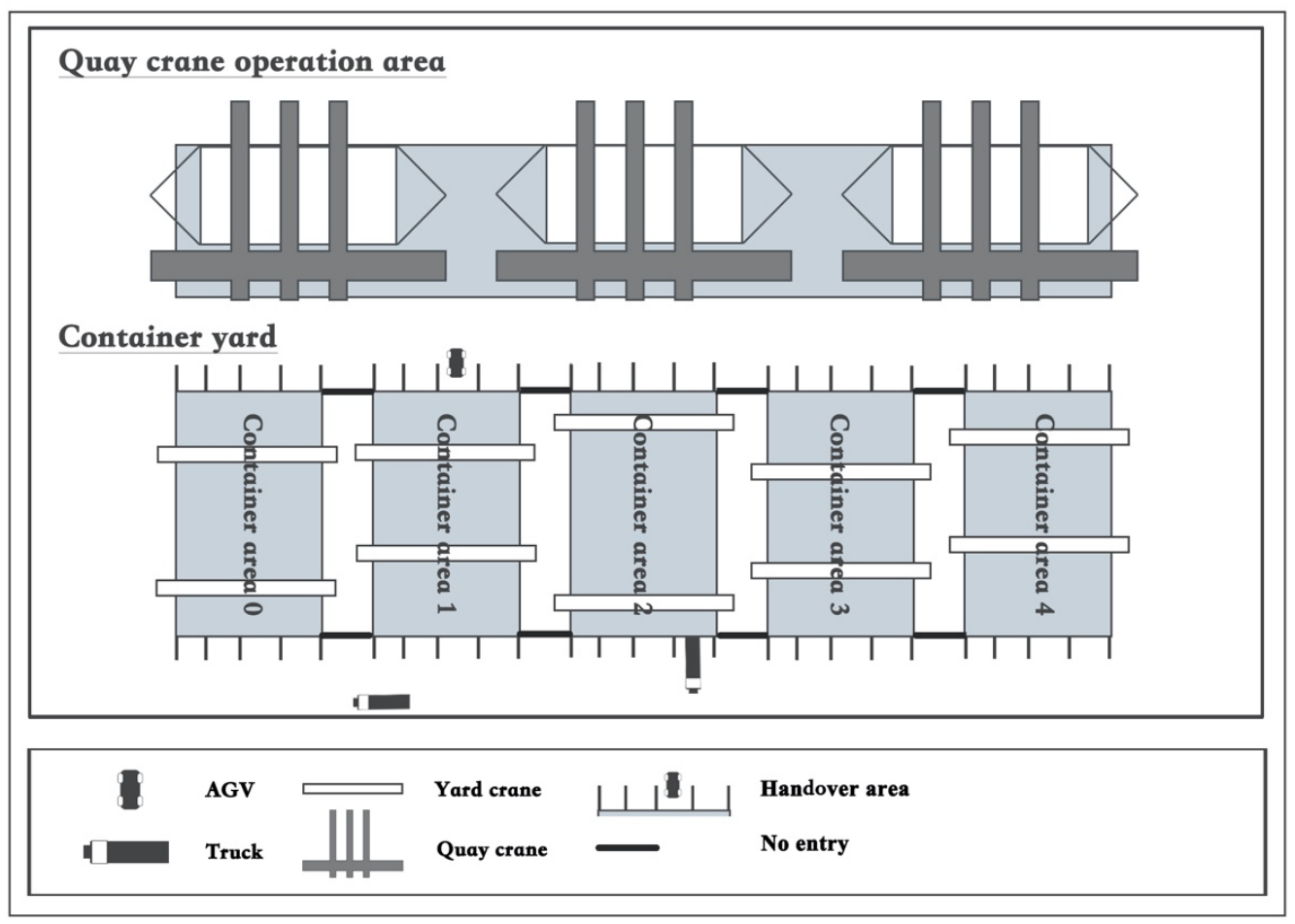

Figure 1. Traditional automated terminal.

The new U-shape trafficked automated terminal is proposed by Shanghai Zhenhua Heavy Industries Co., Ltd. (ZPMC) [1]. It was applied in the Qinzhou Port of Beibu Gulf Port Co., Ltd. [2]. Figure 2 shows the layout of the new U-shape trafficked automated terminal [3]. It is mainly optimized for the yard. Different from the previous handover mode, the U-shape trafficked automated terminal adopts dual cantilever rail cranes as YCs. There are no handover areas at the ends of the yard. YCs' handover is with AGVs and ETs on both sides of the block, respectively, avoiding long-distance and heavy-load transporting of containers. The AGV and ET have separate lanes. The AGV and ET come from/to the yard along the U-shaped lanes and hand over containers with YC. After the handover work is completed, ET can leave the port directly. Two adjacent storage yard blocks share AGV or ET lanes, and YC can be directly handed over with AGV and ET at both ends of the cantilever. The U-shape trafficked layout is aimed to avoid the problems of traditional terminal by optimizing the handover mode among YC, AGV and ET: (1) YC adopts a structure with a spreader on both ends, and AGVs and ETs can reach the designated position to hand over with $Y C$, avoiding long-time long-distance, heavy-load container handling of YC; (2) the transportation roads of AGVs and ETs are completely separated, so there is no longer a mixed congestion problem of AGV and ET; and (3) and ET can leave the port directly after its handover is completed, which improves the transportation efficiency of the overall operation.

The scheduling strategy of the traditional terminal yard is no longer applicable to the yard under the new U-shape trafficked layout. In the traditional handover mode, AGV or ET hands over with YC in the handover area at both ends of the yard. In the U-shape trafficked layout, AGV or ET enters the yard for handover with YC. AGV and ET lanes are separated, so there is no interference between AGV and ET, but there is a new path interference occurring in AGVs or ETs. Significant changes take place in the handover mode of YC, AGV and ET. Because YC can hand over separately with AGV and ET at same bay (parallel to the $Y C$ cart track), we need to consider the mixed constraint between $Y C$, AGV and ET, not just the route interference problem of ET or AGV. Therefore, the existing scheduling schemes of the traditional terminal yard cannot solve the current new problem, which is more complicated and challenging. 


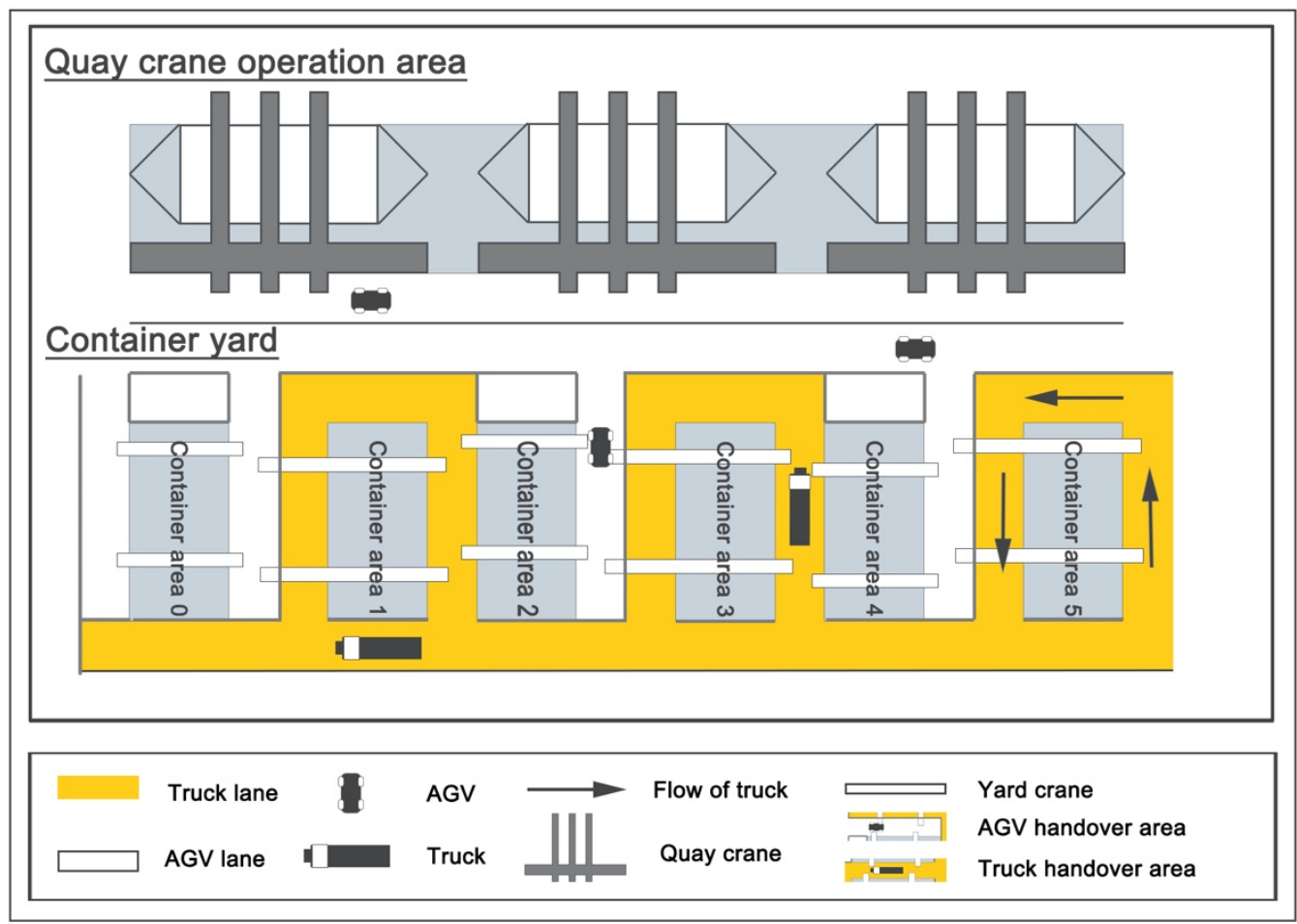

Figure 2. U-shape trafficked automated terminal.

The new U-shape trafficked automated terminal is proposed by ZPMC to improve the efficiency of port operations. The handover mode between YC and AGV or ET has changed, bringing new mixed constraints. A new hybrid scheduling problem has arisen in the new U-shaped trafficked automated terminal, which is more complex and harder. In this study, we propose a static and dynamic mixed scheduling strategy to solve the problem, and we abstract the attributes contained in the scheduling object (such as the task scheduling parameters of $\mathrm{YC}, \mathrm{AGV}$ and ET; the start time and end time of each task; the total task completion time; and the tasks corresponding to the queue at the yard entrance) to form a module. We connected these modules to build a new model architecture. This architecture makes it more convenient to modify and optimize the hybrid scheduling problem. To improve PSO, a chaos particle swarm optimization with speed control (CCPSO) is proposed. In addition, we propose a mapping space from decision variables to particles to solve the contradiction between algorithm iteration and model solving.

The rest of the paper is organized as follows: Section 2 reviews the relevant literature. Section 3 introduces the multi-equipment scheduling layered model structure and develops the hybrid scheduling problem model. Section 4 provides the details of CCPSO, which includes an improvement of particle mapping space, particle chaos strategy and particle speed control strategy for CCPSO. In Section 5, numerical simulation experiments are carried out to verify the effectiveness of the proposed algorithm, and PSO, APSO and RPPSO are used to compare with CCPSO in different container sizes, AGV numbers. The last section gives the conclusion and future research direction.

\section{Literature Review}

YC, QC, AGV and ET are all common dispatching machines in container terminals. There has been a lot of research aimed at improving the efficiency of these loading and unloading transportation machines. Iris, C. et al. [4] reviewed some innovative technologies in improving port energy efficiency and pointed out that there is a great potential for ports to achieve further energy efficiency, and researchers have many impactful research opportunities. Most of the scheduling research is aimed at the scheduling of a single equipment. Some researchers have studied the QC scheduling, and they mainly solve the 
container loading and unloading sequence of the QC [5,6] A large number of researchers have studied the YC scheduling. The non-interference constraint of double YCs and the consideration of multiple environmental variables are the research focus of these problems [7-13]. For AGV scheduling problem, most researchers focus on finding the optimal vehicle allocation and path planning $[14,15]$.

In general, container transportation tasks require the cooperation of multiple resources (such as cranes and vehicles). Separate scheduling of various equipment resources may lead to suboptimal solutions. If there is no proper and efficient schedule (result of container transportation sequences for equipment), waiting time increases, resulting in lost productivity and increased costs. At present, there are still only a few papers on the study of terminal multi-equipment scheduling, especially when three or more kinds of equipment are involved. Iris, C. et al. [16] researched the ship loading problem with transfer vehicle considered. For AGV and YC scheduling problems, Chen, X. et al. [17] designed a task assignment mode under multiple AGVs and YCs scheduling, in which the YC handover area is at the side of the container yard. The AGV path constraint and YC no-crossings interference are considered. To solve these problems effectively, they drew on some research results in the field of multi-robot task assignment problem (MRTA). Based on the construction of AGV space-time network diagram, they used the Alternating Directional MultiPLication (ADMM) algorithm to make the solution approach to the optimal solution better through linear direction coefficient. In QC and AGV scheduling, Houming, F. et al. [18] considered time- and energy-consumption optimization. The energy consumption objectives include the energy consumption of QC operation and the waiting energy consumption caused by disturbance. Due to the interaction between the two objective functions of energy and time consumption, the near optimal solution could only be achieved in accordance with the actual situation. Iris, C. et al. [19] used a robust method for QC scheduling, considering ship loading problem. At present, researches mainly focus on one or two kinds of terminal equipment dispatching, while researches involving more than three kinds of equipment dispatching are relatively few. He, J. et al. [20] established a solving model by integer programming, in which the energy consumption is taken as objective function. They established the fitness function by classifying the energy consumption of QC, terminal truck (TC) and AGV in different motion states. Then the genetic algorithm and PSO algorithm were used to solve the problem. However, their solving model ignored the AGV, TC path interference constraints and the difference between time-consuming of $Q C$ and $Y C$. Moreover, the local optimal problem was not resolved well.

To sum up, the current research on terminal multi-equipment scheduling generally focuses on the scheduling between two kinds of equipment at most, such as AGV and YC, AGV and QC, YC and internal truck (IT), etc. However, the interaction among YC, AGV and ET at the U-shape trafficked automated terminal is much closer than that at traditional automated container terminals. Constraints among three kinds of equipment, such as the path constraint of AGV and ET, and the no-crossing constraint of QC or YC, should be considered. This paper studies the hybrid scheduling for $\mathrm{YC}, \mathrm{AGV}$ and ET at the U-shape trafficked automated terminal, considering the quantity allocation of AGV and path constraint of AGV and ET. CCPSO which combines both advantages of chaos search and particle swarm optimization is proposed for the hybrid scheduling. The algorithm not only converges quickly, but also can effectively avoid the precocity problem.

\section{Mathematical Model}

\subsection{Problem Description}

Figure 3 shows one yard unit of the U-shape trafficked terminal. Each unit contains two blocks. Each block is equipped with 2 YC with spreader on both ends structure, which is responsible for handover with AGV and ET passing by the side of the yard. Before the AGV or ET enters the yard, it needs to queue up and wait in turn at the yard lane entrance. Each AGV or ET enters and exits the yard along the U-shape trafficked route marked in Figure 3. We also have the following assumptions: 
- $\quad \mathrm{AGV}$ and ET lanes are one-way roads.

- The YC cart running 1 bay consumes one time unit.

- The speed ratio of the YC spreader empty running, the YC spreader full-load running, the $Y C$ trolley running, the $Y C$ cart empty running and the $Y C$ cart full-load running is 1:0.5:0.3:1:0.25 (the speed parameters reference those at Qinzhou Port).

- This study mainly focused on the loading and unloading operations of terminal yard.

- We assume that ET is abundant and one ET is only responsible for one container.

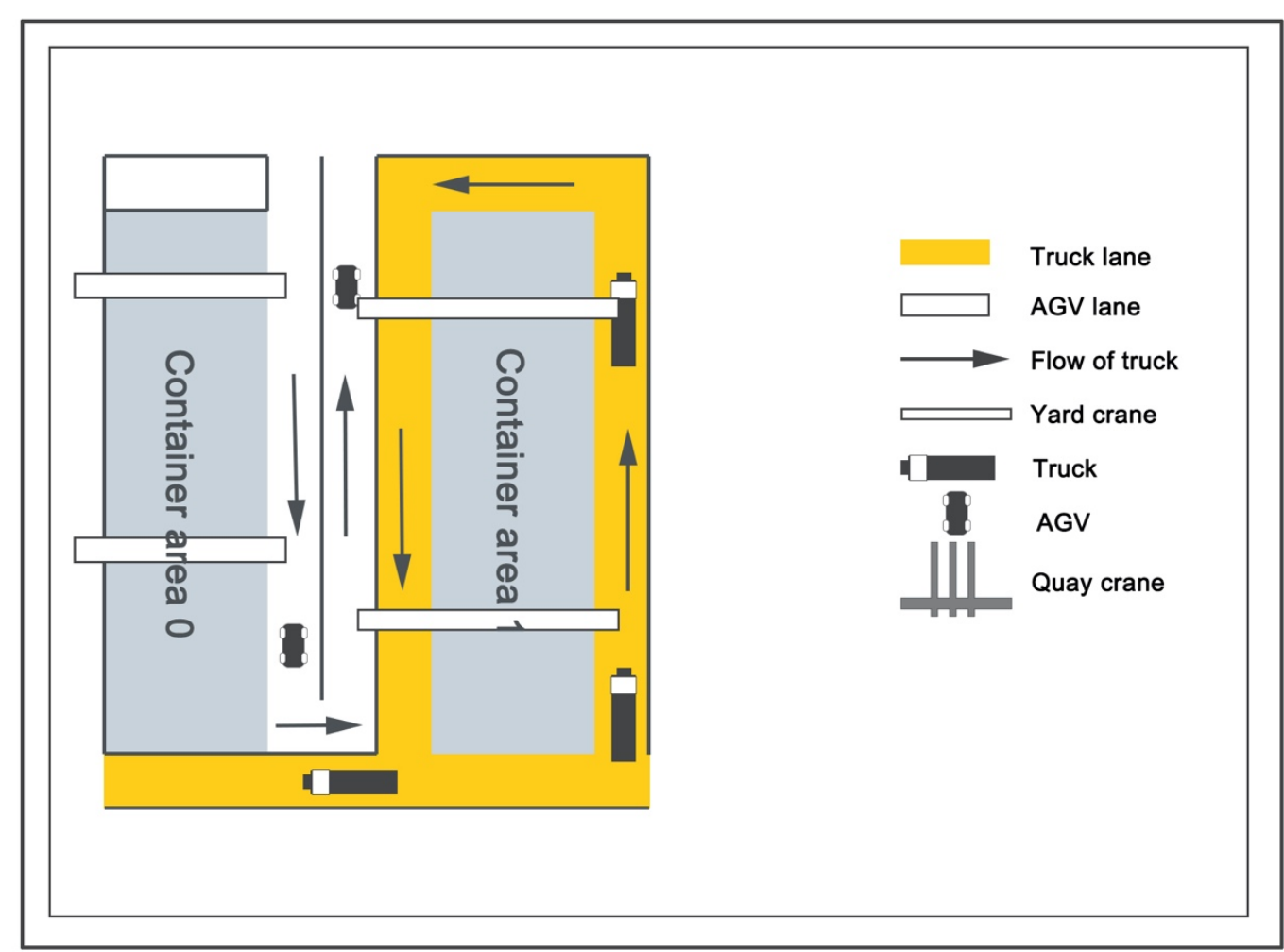

Figure 3. U-shape trafficked container yard layout.

Decision variable can be represented as follows, where two AGVs, two ETs and four YCs are allocated to transport five containers; and 0 and $0^{*}$ represent the virtual start and end tasks, respectively:
AGV1: $0 \rightarrow 1 \rightarrow 0^{*}$.
AGV2: $0 \rightarrow 3 \rightarrow 2 \rightarrow 0^{*}$.
YC1: $0 \rightarrow 2 \rightarrow 0^{*}$.
YC2: $0 \rightarrow 3 \rightarrow 0^{*}$.
YC3: $0 \rightarrow 5 \rightarrow 4 \rightarrow 0^{*}$.
YC4: $0 \rightarrow 1 \rightarrow 0^{*}$.
ET1 2: $0 \rightarrow 5 \rightarrow 4 \rightarrow 0^{*}$.

\subsection{Notations}

We introduce the following notations as Tables 1 and 2:

Table 1. Parameter notations.

\begin{tabular}{cl}
\hline Parameters & \multicolumn{1}{c}{ Notations } \\
\hline $\mathrm{U}$ & $\mathrm{YC}, \mathrm{AGV}$ and ET, indexed by $u \in\{0,1,2\}$. \\
$\mathrm{YC}_{i_{0}}$ & Set of YC, indexed by $i_{0} \in\{1,2,3, \ldots, Y c\}$. \\
$\mathrm{ET}_{i_{1}}$ & Set of ET, indexed by $i_{1} \in\{1,2,3, \ldots, E t\}$. \\
\hline
\end{tabular}


Table 1. Cont.

\begin{tabular}{|c|c|}
\hline Parameters & Notations \\
\hline $\mathrm{AGV}_{i_{2}}$ & Set of AGV, indexed by $i_{2} \in\{1,2,3, \ldots, A g v\}$ \\
\hline $\mathrm{J}_{i_{\mathrm{u}}}$ & $\begin{array}{c}\text { Set of equipment task number, indexed by } i_{u}, j \in\left\{\mathrm{J}_{i_{u}}^{0}, 1,2,3, \ldots, \mathrm{J}_{i_{u}}, \mathrm{~J}_{i_{u}}^{e}\right\} \cdot \mathrm{J}_{i_{u}}^{0} \text { : } \\
\text { the virtual initial task; } \mathrm{J}_{i}^{e} \text { : the virtual final task. }\end{array}$ \\
\hline$b_{n}$ & Bay where container $n$ should be located. \\
\hline$r_{n}$ & Row where container $n$ should be located. \\
\hline$r_{a g v}$ & Handover row of AGV. \\
\hline $\mathrm{r}_{e t}$ & Handover row of ET. \\
\hline $\mathrm{f}_{n}$ & The descent distance of the YC spreader when transporting container $n$. \\
\hline $\mathrm{f}_{0}$ & The descent distance of YC spreader during handover. \\
\hline$d_{n}$ & Task type of container $n$ \\
\hline $\mathrm{B}$ & Set of yard lane, indexed by $\beta \in\{-1,0,1, \ldots, B\} .-1$ : entrance of yard lane \\
\hline $\mathrm{y}_{n}$ & $\begin{array}{l}\text { Block number of container } n ; 0 \text {, block with AGV lane on right hand; } 1 \text {, block } \\
\text { with ET lane on right hand. }\end{array}$ \\
\hline $\operatorname{sort}(x, y)$ & $\begin{array}{l}\text { The function is to adjust the size order of the elements in } x \text { to correspond to the } \\
\text { size order of the elements in y. For example: } x=(0,1,2), y=(5,4,3) \text {, according to } \\
\text { the order of the element size in } y, x \text { is adjusted to }(2,1,0) \text {. }\end{array}$ \\
\hline $\max (x, y)$ & The maximum value of $x$ and $y$ \\
\hline
\end{tabular}

Table 2. Variable notations.

\begin{tabular}{c} 
Notations \\
\hline $\begin{array}{c}\text { Variables } \\
y_{i j} \\
a_{i j}\end{array}$ \\
$\begin{array}{c}\text { Maximum completion time. } \\
e_{i j}\end{array}$ \\
$\begin{array}{c}\text { Container number of } Y C i, \text { task } j . \\
\text { Container number of AGV } i, \text { task } j .\end{array}$ \\
Container number of ET $i$, task $j$.
\end{tabular}

\subsection{Layered Architecture}

The container terminal scheduling model becomes more and more complex with the increase of container transportation and it is harder to read and understand the scheduling model with constraints increasing. When the considered dynamic variables increase, the scheduling model needs to be re-established, which brings workload. In addition, it brings difficulty in combination of the model and the algorithm. A layered architecture is proposed to optimize the hybrid scheduling model. Each layer of the architecture consists of several modules, and each module consists of several abstract sub-blocks. The abstract sub-blocks become an entity object by setting real value. The logical relationships between modules are determined by the topmost architecture content, so local changes within modules do not affect the whole. The new architecture can capture the key features of problem, and improve the research efficiency. 
As shown in Figure 4, the model is firstly divided into four levels: initialization layer, circulation layer, particle layer and scheduling layer. In the initialization layer, the general task information is used to initialize the particle swarm in the loop body. In the circulation layer the loop body contains the algorithm iteration logic. Both the initialize layer and the circulation layer belong to the outer framework of the model. The outer framework is mainly responsible for the initialization of the abstract module, as well as the algorithm cycle for iteration and calculation. The inner architecture includes the particle swarm layer and the scheduling layer. The particle swarm layer includes the algorithm method (particle iteration model and chaos model in Figure 4), and the swarm module is an abstraction of the particle swarm, the abstract particle module can be objectified through this part. The particle swarm parameters generally include the total number of particles, the velocity of each particle, the local historical optimal position, the global optimal position, the global optimal value and the local historical optimal value. In the scheduling layer, scheduling module belongs to the abstraction of the single decision variable of scheduling problem, and it includes the scheduling parameters and fitness value solving model. In this paper, the scheduling parameters include the fitness value and the decision variables (scheduling arrangements of $Y C, A G V$ and ET). After the model initialized, the scheduling parameters of the scheduling module become real objects. When the fitness value solving model is called by the outer structure, the dynamic tracking model of the lane occupancy information, the queuing model and the time update model are used to solve the completion time of current decision variables. The solving value is then assigned to the fitness value.

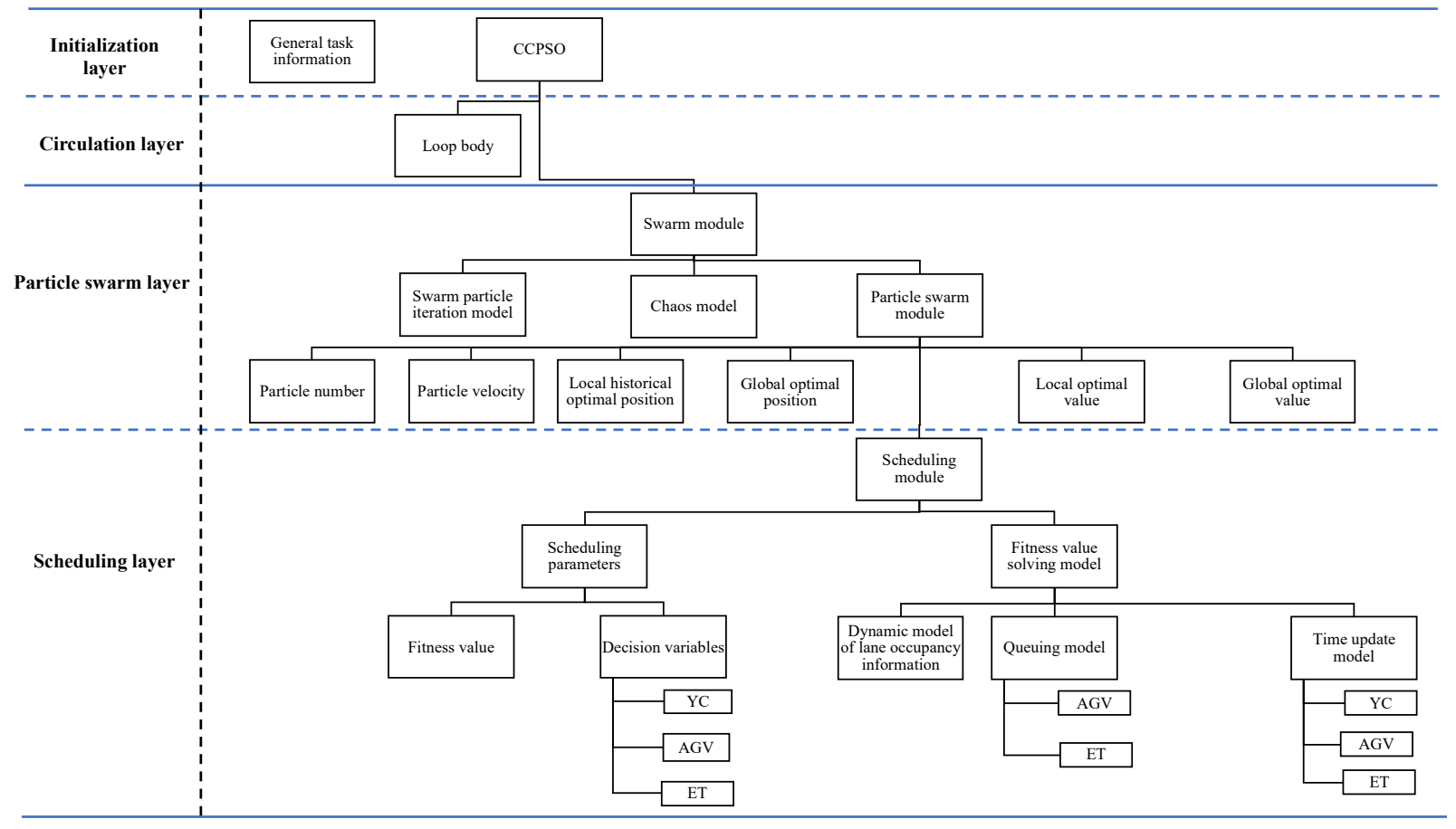

Figure 4. Layered architecture.

3.4. Model

3.4.1. Objective Function

$$
\min F=\max \left(y t_{i_{0} e}, e t_{i_{1} e}, a t_{i_{2} e}\right)
$$

Objective Function (1) is used to minimize the overall maximum completion time, F, which depends on the final completion time of the transportation equipment (YCs, AGVs and ETs). 


\subsubsection{Static and Dynamic Mixed Scheduling Strategy}

Shyalika et al. [21] mentioned that the scheduling strategies in current research are mainly divided into static scheduling and dynamic scheduling. In static scheduling, the decision variables (task assignment) are assumed to be known in the computation. In dynamic scheduling, the decision variables are unknown in the computation, and many parallel strategies are generated in the computation project until the last task is assigned.

As shown in Figure 5, in the U-shape trafficked terminal, one YC task's completion time depends on the arrival time of AGV or ET at the handover area. Meanwhile, to satisfy the path interference constraint, there is a waiting time as AGV or ET is waiting until other AGVs or ETs on its path are leaving. Thus, the arrival time of AGV or ET to the handover area is affected by other AGVs or ETs. When arriving at the handover area, AGV or ET waits for YC to complete its handover work. YC, AGV and ET are mutually restrictive when transporting containers in the yard. Therefore, the coupling relationship between YC, AGV and ET in the U-shape trafficked terminal yard is described as a mixed constraint problem. Since the previous static strategy is not able to solve the problem, static strategy is combined with dynamic strategy in this study. This strategy tracks road occupancy information by assuming that the decision variables are known, and it uses a dynamic solution to solve the fitness value, that is, the maximum completion time.

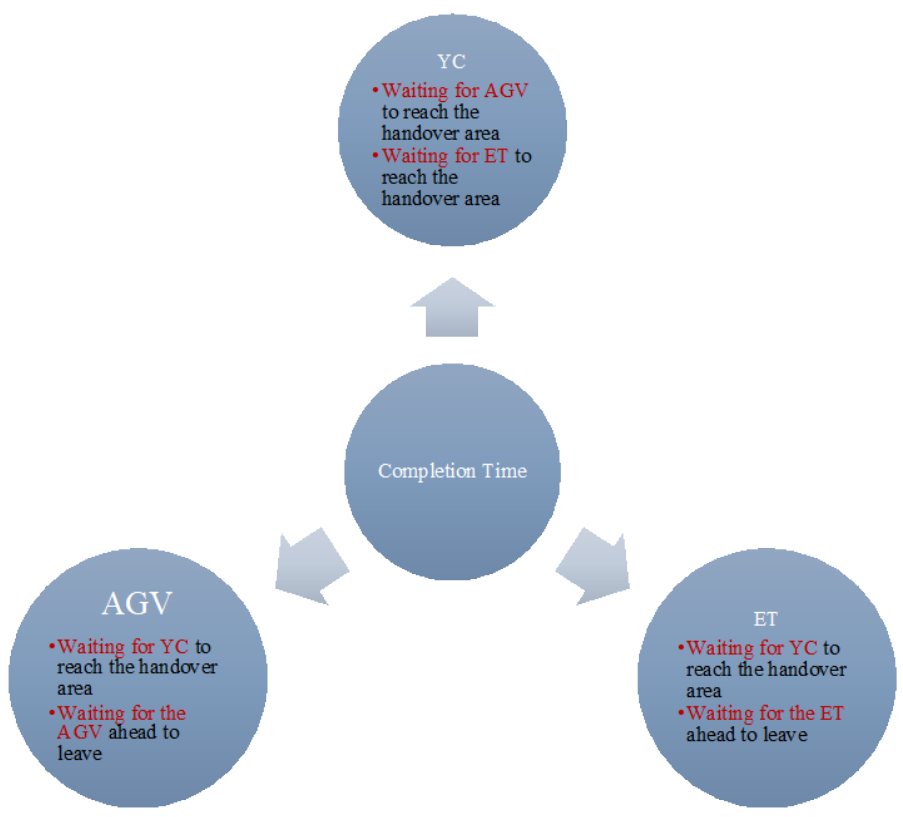

Figure 5. Constraint relationship of $Y C, A G V$ and ET.

(a) Static Scheduling

The algorithm loop is based on known decision variables. The tasks information including the container number, the task type corresponding to the container, the target location of the container in yard is input to initialize the computation. Each container has a unique numerical number. The corresponding tasks of each container are divided into four types as shown in Table 3 below. Type 1 indicates that export containers are transported out of the yard through $Y C$ and AGV in turn. Type 2 indicates that containers are transported from AGV to the yard, and then from $Y C$ to the target location of the corresponding yard. The transportation modes of types 3 and 4 are similar to types 1 and 2, respectively. For types 1 and 3, YC moves to the target location of the yard to get the container first, and then it waits until AGV or ET arrives at the handover area. The current task is completed after $Y C$ finishes the handover. For task type 2 and 4, the YC trolley first moves to the handover row of target bay and then waits for the handover with AGV or ET. Then YC only needs the movement of the trolley and spreader to transport the container to the target location at the current yard location. 
Table 3. Task type.

\begin{tabular}{ccc}
\hline Task Type & Equipment 1 & Equipment 2 \\
\hline 1 & YC & AGV \\
2 & AGV & YC \\
3 & YC & ET \\
4 & ET & YC \\
\hline
\end{tabular}

AGVs or ETs need to line up at the entrance of the yard first, and enter or leave when there are no other AGVs or ETs on path. The AGV that first hands over with YC at the yard has priority. At the handover area, YC, AGV or ET need to wait until the corresponding handover equipment arrives. The completion time of the current task is impacted by the transportation and handover time of the transportation equipment, and the completion time of the previous task. Thus, there is a coupling relationship between the above problems. In addition to solving the hybrid scheduling problem of $Y C$, AGV and $\mathrm{ET}$, it is necessary to access their status in real time.

\section{(b) Dynamic Scheduling}

Since the bay is parallel to the lane at the yard, the bay position can be corresponded to the lane coordinates one to one. Through the occupancy status of each location in yard lanes, the information of AGV or ET entering the yard can be tracked in real time. As shown in Table 4 whether AGV or ET can move is decided by the lane occupancy information. If the lane coordinates are released, the location can be passed. When one AGV or ET waits on the lane, the release time of the corresponding coordinate position is renewed.

Table 4. Lane tracking.

\begin{tabular}{ccc}
\hline Lane Coordinates & Release Time (AGV Lane) & Release Time (ET Lane) \\
\hline-1 & 0 & 0 \\
0 & 2 & 15 \\
1 & 10 & 3 \\
2 & 5 & 9 \\
$\ldots$ & $\ldots$ & $\ldots$ \\
\hline
\end{tabular}

When the value of decision variable is initialized, the dynamic scheduling starts until the solving the fitness value and update the decision variable to satisfy all the constraints. When the current container is transported by AGV or ET, the dynamic process and the information updated in process are shown in Figure 6. At each iteration of the dynamic scheduling, all YC tasks are traversed one by one. When all the tasks of a YC are performed, the $Y C$ is skipped, and the dynamic process stops until all the $Y C$ tasks are traversed. As one YC task is traversed, the AGV or ET transporting the same container is lined up at entrance of queue, and the container numbers and arrival times of AGVs or ETs in queue are updated. Then the AGV or ET at the top of the queue enters the yard at the time the entrance lane released. Through the road occupancy information, we can know whether there are other AGVs or ETs on path. Moreover, the road occupancy information is updated once an AGV or ET arrives, waits or leaves. At the handover process, the start time of the YC task may be updated if the AGV or ET arrives at the handover position later than the YC. After the AGV or ET leaves, the designed task pointer for the current $Y C$ continues to track the subsequent $Y C$ tasks until all YC tasks are completed.

Constraint (2) defines the initialization of $y t_{i j}$ where AGV and ET interference is not taken into account. In the following dynamic scheduling, $y t_{i j}$ is updated continuously, until all the constraints are met. 


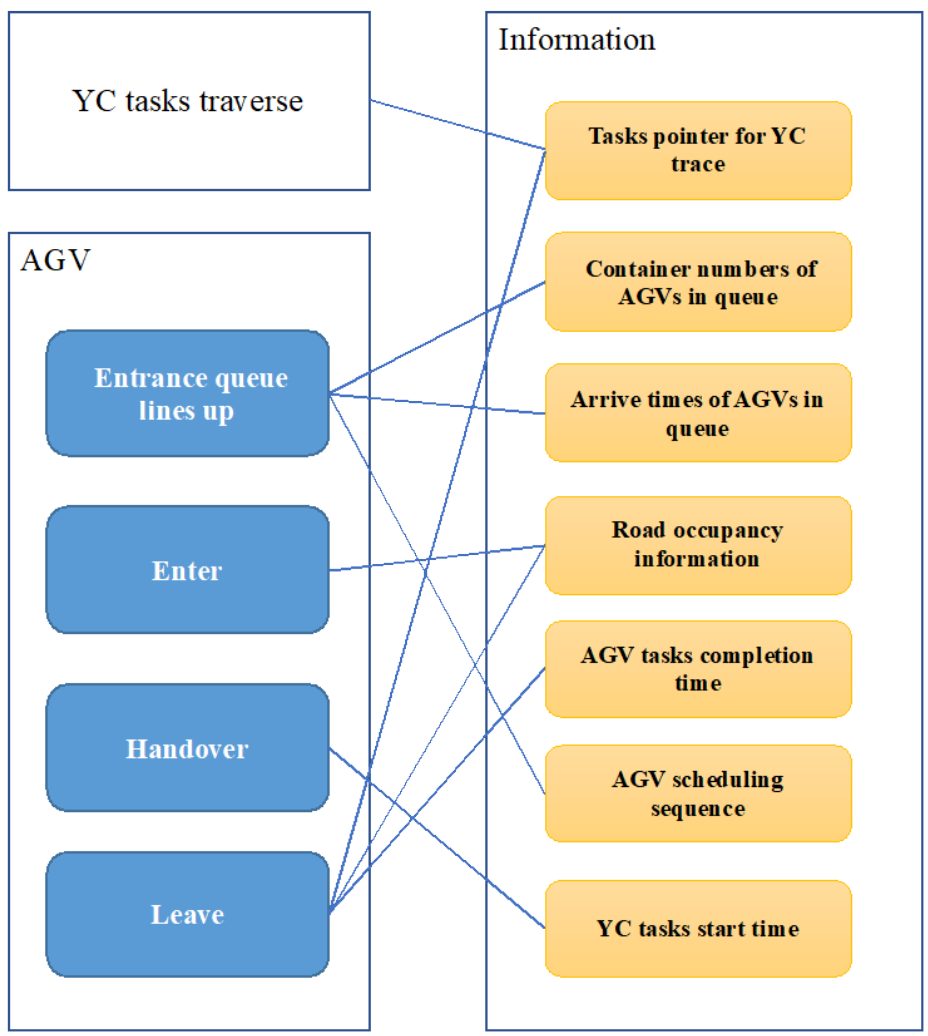

Figure 6. Dynamic process.

Constraint (3) defines the update of the container number of the current YC task $S_{i}^{n}$. When all of the YC tasks are traversed, $S_{i}^{n}$ is set to 0 as a placeholder. Constraint (4) defines the update of $S_{i}^{t}$ when $Y C i$ arrives at the handover area.

$$
\begin{aligned}
& y t_{i j+1}=\left\{\begin{array}{c}
y t_{i j+1}+\max \left(\frac{\left|b_{y_{i j}}-b_{y_{i j-1}}\right|}{0.25}+\frac{\left|r_{a g v}-r_{y_{i j-1}}\right|}{0.3}\right)+\frac{\left|r_{a g v}-r_{y_{i j}}\right|}{0.3}+3\left(f_{y_{i j}}+f_{0}\right), d_{y_{i j}} \in\{1,2\} \\
y t_{i j+1}+\max \left(\frac{\left|b_{y_{i j}}-b_{y_{i j-1}}\right|}{0.25}+\frac{\left|r_{y_{i j}}-r_{y_{i j-1}}\right|}{0.3}\right)+\frac{\left|r_{e t}-r_{y_{i j}}\right|}{0.3}+3\left(f_{y_{i j}}+f_{0}\right), d_{y_{i j}} \in\{3,4\}
\end{array}\right. \\
& S_{i}^{n}=\left\{\begin{array}{l}
0, j \geq \mathrm{J}_{i_{0}} \\
y_{i j}, \text { other }
\end{array}\right. \\
& S_{i}^{t}=\left\{\begin{array}{c}
y t_{i j}+\max \left(\frac{\left|b_{y_{i j}}-b_{y_{i j-1}}\right|}{0.25}+\frac{\left|r_{y_{i j}}-r_{y_{i j-1}}\right|}{0.3}\right)+3 f_{y_{i j}}+\frac{\left|r_{a g v}-r_{y_{i j}}\right|}{0.3}, d_{y_{i j}}=1 \\
y t_{i j}+\max \left(\frac{\left|b_{y_{i j}}-b_{y_{i j-1}}\right|}{0.25}+\frac{\left|r_{y_{i j}}-r_{y_{i j-1}}\right|}{0.3}\right)+3 f_{y_{i j}}+\frac{\left|r_{e t}-r_{y_{i j}}\right|}{0.3}, d_{y_{i j}}=3 \\
y t_{i j}+\max \left(\frac{\left|b_{y_{i j}}-b_{y_{i j-1}}\right|}{0.25}+\frac{\left|r_{a g v}-r_{y_{i j-1}}\right|}{0.3}\right), d_{y_{i j}}=2 \\
y t_{i j}+\max \left(\frac{\left|b_{y_{i j}}-b_{y_{i j-1}}\right|}{0.25}+\frac{\left|r_{e t}-r_{y_{i j-1}}\right|}{0.3}\right), d_{y_{i j}}=4
\end{array}\right. \\
& \mathrm{A}=\mathrm{A}+\left\{y_{i_{0}^{\prime} j_{0}^{\prime}}\right\} \\
& \mathrm{A} t=\mathrm{A} t+\left\{a t_{i j}\right\}, \forall a_{i j}=y_{i_{0}^{\prime} j_{0}^{\prime}} \\
& a=\operatorname{sort}\left(\mathrm{a}, \max \left(A t, S_{i}^{t}\right)\right), \forall i \in\left\{1,2,3, \ldots, Y_{c}\right\} \\
& i_{1}=i, j_{1}^{\prime}=j, \forall a_{i j}=\mathrm{A}_{0}
\end{aligned}
$$




$$
\begin{gathered}
j_{1}=j, \forall a t_{i_{1} j-1} \neq 0, a t_{i_{1} j}=0 \\
a_{i_{1} j}=\left\{\begin{array}{c}
\mathrm{A}_{0}, j=j_{1} \\
a_{i_{1} j-1}, j \in\left(j_{1}, j_{1}^{\prime}\right] \\
a_{i_{1} j}, j \in\left(j_{1}^{\prime}, \mathrm{J}_{i_{1}}\right]
\end{array}\right. \\
t_{\text {enter }}=\max \left(a t_{i_{1} j_{1}}, Y_{-1}^{0}+0.1\right) \\
t_{\text {handover }}=\max \left(t_{\text {enter }}, Y_{\beta}^{0}+\Delta \mathrm{t}_{0}\right), \forall \beta \leq b_{\mathrm{A}_{0}} *\left(y_{\mathrm{A}_{0}}+1\right) \\
Y_{-1}^{0}=t_{\text {handover }} \\
i_{0}=i, j_{0}=j, \forall y_{i j}=\mathrm{A}_{0} \\
y t_{i_{0} j_{0}}=y t_{i_{0} j_{0}}+t_{t_{\text {handover }}}-S_{i_{0}}^{t}, \forall t_{\text {handover }}>S_{i_{0}}^{t} \\
t_{\text {wait }}=\max \left(t_{\text {handover }}, S_{i_{0}}^{t}\right)+3 f_{0} \\
t_{\text {leave }}=\max \left(t_{\text {wait }}, Y_{\beta}^{0}+\Delta \mathrm{t}_{0}\right), \forall \beta \leq B \\
Y_{\beta}^{0}=t_{\text {leave }}, \forall \beta=b_{\mathrm{A}_{0}} *\left(y_{\mathrm{A}_{0}}+1\right) \\
\text { at } t_{i_{1} j_{1}+1}=t_{\text {leave }}+\Delta \mathrm{t}_{1} \\
\mathrm{~A}=\mathrm{A}-\left\{\mathrm{A}_{0}\right\}
\end{gathered}
$$

Constraints (5) to (20) define the dynamic update process when $y_{i^{\prime} 0 j^{\prime} 0}$ is transported by AGV. When there is an YC for a new task and this task's container is also transported by the AGV, the task information of AGV queue at entrance is updated. The new arrival is put at the end of the AGV queue. Constraints (5) and (6) define the update of AGV queue's information at the yard entrance, which includes A and At. Constraints (7) define the queuing priority. The earlier the AGV and its corresponding $Y C$ arrive at the entrance and handover area, the higher priority the AGV has. Each time an AGV enters the queue, the AGV queue at the yard entrance is rearranged according to the above priority. Constraint (8) and (9) defines AGV number $i_{1}$ and task number $j_{1}$ of the top AGV at the current AGV queue. Since the AGV container task sequence changes after the re-queuing, the AGV scheduling order also needs to be updated according to constraint (10). Constraints (11) to (19) define the update of $a t_{i_{1}}, y t_{i j}$ and the release time of the position $Y_{\beta}^{0}$ on handover lane. Constraints (11), (12) and (17) define the entry, handover and departure time of AGV with task $a_{i_{1} j_{1}}$, considering that the path interference (AGV or ET can move when there is no AGV or ET on its path). Constraint (11) defines the entry time of AGV $i_{1}$, which is decided by the release time of AGV lane' entrance and the arrival time of AGV $i_{1}$. Constraint (12) defines the time AGV $i_{1}$ arriving at the handover area, $\Delta \mathrm{t}_{0}$ is a constant coefficient used for collision avoidance. Constraint (13) defines the update of release time of AGV lane's entrance. Constraint (14) defines the YC number $i_{0}$ and task number $j_{0}$ responsible for the transportation. As mentioned above, $y t_{i_{0} j}$ is initialized first, as AGV and ET constraints are not taken into account. Constraint (15) defines the update of $y t_{i_{0} j}$ when YC $i_{0}$ need to wait AGV $i_{1}$ at the handover area. Constraint (16) defines the waiting time of AGV $i_{1}$ at the handover area. Constraint (17) defines the departure time of AGV $i_{1}$. Constraint (18) defines the update of the lane release time after AGV $i_{1}$ leaves. Constraint (19) defines the update of the completion time of AGV $i_{1}$ task $j_{1}$ (the completion time of the task is defined as the start time of the next task). $\Delta t_{1}$ is a constant coefficient which describes how long the AGV spends until it reaches the entrance of the yard next time. Constraint (20) defines the update of the AGV queue at the entrance.

$$
\begin{gathered}
E=E+\left\{y_{i_{0}^{\prime} j_{0}^{\prime}}\right\} \\
E t=E t+\left\{e t_{i j}\right\}, \forall e_{i j}=y_{i_{0}^{\prime} j_{0}^{\prime}}
\end{gathered}
$$




$$
\begin{gathered}
e=\operatorname{sort}\left(e, \max \left(E t, S_{i}^{t}\right)\right), i \in\left\{1,2,3, \ldots, Y_{C}\right\} \\
i_{2}=i, j_{2}^{\prime}=j, \forall e_{i j}=E_{0} \\
j_{2}=j, \forall e t_{i_{2} j-1} \neq 0, e t_{i_{2} j}=0 \\
e_{i_{2} j}=\left\{\begin{array}{c}
E_{0}, j=j_{2} \\
e_{i_{2} j-1}, j \in\left(j_{2}, j_{2}^{\prime}\right] \\
e_{i_{2} j}, j \in\left(j_{2}^{\prime}, \mathrm{J}_{i_{2}}\right]
\end{array}\right. \\
t_{\text {enter }}=\max \left(e t_{i_{2} j_{2}}, Y_{-1}^{1}+0.1\right) \\
t_{\text {handover }}=\max \left(t_{\text {enter }}, Y_{\beta}^{1}+\Delta \mathrm{t}_{0}\right), \forall \beta \leq b_{E_{0}} \\
Y_{-1}^{1}=t_{\text {handover }} \\
i_{0}=i, j_{0}=j, \forall y_{i j}=\mathrm{E}_{0} \\
y t_{i_{0} j_{0}}=y t_{i_{0} j_{0}}+t_{\text {handover }}-S_{i_{0}}^{t}, \forall t_{\text {handover }}>S_{i_{0}}^{t} \\
t_{\text {wait }}=\max \left(t_{\text {handover }}, S_{i_{0}}^{t}\right)+3 f_{0} \\
t_{\text {leave }}=\max \left(t_{\text {twait }}, Y_{\beta}^{1}+\Delta \mathrm{t}_{0}\right), \forall \beta \leq B \\
Y_{\beta}^{1}=t_{\text {leave, }}, \forall \beta=b_{E_{0}} \\
e t_{i_{2} j_{2}+1}=t_{\text {leave }}+\Delta \mathrm{t}_{1} \\
E=E-\left\{E_{0}\right\}
\end{gathered}
$$

Constraints (21) to (36) define the dynamic update process when container is transported by ET. The update process of ET is similar to that of AGV. It should be noted that ET can only hand over with YC in the first half of the U-shape trafficked lane, while AGV can hand over with the YCs of different blocks in the first half and the second half of the AGV lane.

\section{The Algorithm}

\subsection{Particle Mapping Space}

Scheduling decision variables are generally represented by integers, but integer particles are not conducive to iteration. Therefore, continuous mapping of decision particles is required in algorithm iteration. Most researchers simply map the elements in decision variables directly to the 0 to 1 space [22,23]. On this basis, some researchers used the roulette method to improve [20]. In the iteration, the decision variables in the form of integer are used in the fitness function of the scheduling problem. Therefore, the particles in the real number domain need to be discretized. To ensure the consistency between the decision variables and the particles, researchers often arrange the order of the size of the elements in the particles according to a fixed order of decision values. However, this brings some problems. Figure 7 shows a group of two-element particles. The particle distribution space is divided by the dotted line $\mathrm{x} 0=\mathrm{x} 1$. It can be seen that $\mathrm{x} 0<\mathrm{x} 1$ below the dotted line, while $\mathrm{x} 0>\mathrm{x} 1$ the elements above the dotted line. When the particles are mapped to decision variables in the order of element size, the upper and lower parts of the dashed line are only mapped to two decision variable values. After algorithm iteration, the value of the decision variable corresponding to the particle is only related to the arrangement of the element size in the particle. That is, the value of the decision variable is only related to the angle between the particle and each axis, and has nothing to do with the actual element value of the particle. When the above particles are used for iteration, the distribution of the optimal solution diverges along the line. When the dimensionality of the decision variable increases, this feature interferes with the iterative direction correction in the iteration process and affect the final solution result. Therefore, this paper uses the angle between the 
decision variable and each axis as the particle to avoid the above problems. The relevant formulas are (37) and (38).

$$
\begin{gathered}
\theta_{i}=\operatorname{arcos} \frac{X \cdot E_{i}}{|X|} \\
X=\operatorname{sort}(\mathrm{M}, \cos \theta)
\end{gathered}
$$

where $X$ is the decision variable, $\theta$ is the algorithm particle, $\theta_{i}$ is the $i$-th dimension element of $\theta, E$ is the identity matrix with the same dimension as $X, M$ is the reference decision value and $E_{i}$ is the $i$-dimensional vector of $E$.

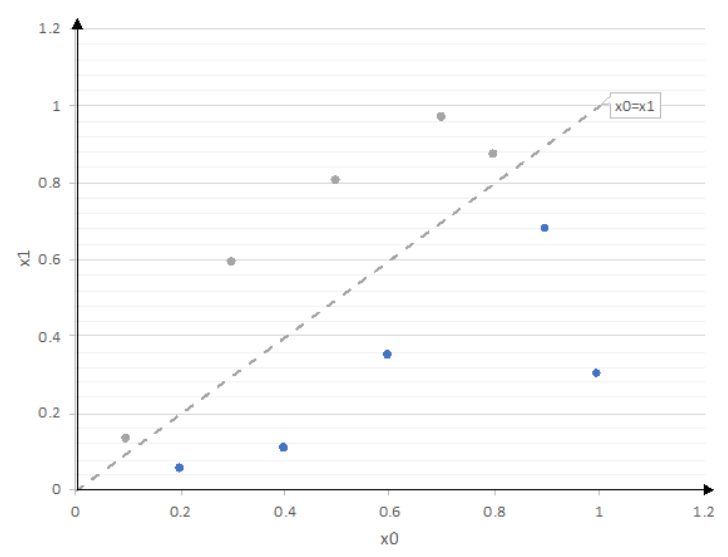

Figure 7. A set of algorithm particles.

Equation (37) represents the $i$-th dimension element of the particle mapped to the decision variable. The element of $\theta$ is composed of the angle between $X$ and its axes (the number of axes is determined by the number of elements contained in $X$ ). Equation (38) represents the process of mapping continuous particles into discrete decision variables. According to the order of the $\cos \theta$ and the decision value $M$ referenced by the size ordering, the decision variable $X$ corresponding to the particle $\theta$ is finally obtained.

An example is shown in Table $5 \mathrm{M}$ is the initial mapping rule, and $X$ is the decision particle. It can be seen that $X$ contains 5 elements, and elements in particle $\theta$ are angles between $X$ and $[1,0,0,0,0],[0,1,0,0,0],[0,0,1,0,0],[0,0,0,1,0],[0,0,0,0,1]$. The index number of $\cos \theta$ sorted from small to large becomes $[5,2,1,4,3]$. According to the reference decision value $M, X$ becomes $[13,9,14,16,1]$ after mapping.

Table 5. The angle mapping.

\begin{tabular}{cccc}
\hline $\mathbf{M}$ & $\boldsymbol{X}$ & $\boldsymbol{\theta}$ (Radian) & $\boldsymbol{c o s} \boldsymbol{\theta}$ \\
\hline 1 & 13 & 1.058 & 0.491 \\
9 & 9 & 1.224 & 0.340 \\
13 & 14 & 0.923 & 0.603 \\
14 & 16 & 1.015 & 0.528 \\
16 & 1 & 1.533 & 0.038 \\
\hline
\end{tabular}

\subsection{Algorithm}

\subsubsection{Particle Velocity Control Strategy}

Traditional particle swarm optimization (PSO) simulates the social behavior of birds in the foraging process. In each iteration, the current iteration velocity of each particle is determined by local optimal position and global optimal position. The velocity and position are normally updated by the following formulae:

$$
\begin{gathered}
v_{i+1}=\omega v_{i}+c_{1} r_{1}\left(\text { pbest }_{i}-p_{i}\right)+c_{2} r_{2}\left(\text { gbest }-p_{i}\right) \\
p_{i+1}=p_{i}+v_{i+1}
\end{gathered}
$$


where $v_{i}$ is the velocity of particle at $i$-th iteration; $\omega$ is the inertia weight; $c_{1}$ is the cognitive coefficient; $c_{2}$ is the social coefficient; $r_{1}$ and $r_{2}$ random numbers in $(0,1)$, regenerated in each iteration; pbest $t_{i}$ is the local best position of particle; and gbest is the global best position of swarm, $p_{i}$ is the position of particle.

In CCPSO, a sort of chaos optimization is added to prevent particles from falling into local optimum, and the search performance of the algorithm is improved by increasing the iterative convergence velocity of particles. The velocity is controlled according to (41)-(43):

$$
\begin{gathered}
\alpha=\frac{v_{i+1} \cdot(p-\text { gbest })}{\left|v_{i+1}\right| * \mid p-\text { gbest } \mid} \\
v_{i+1}{ }^{\prime}=\frac{v_{i+1}}{\left|v_{i+1}\right|} * \mid p-\text { gbest } \mid * \alpha \\
p_{i+1}^{\prime}=p+v_{i+1}{ }^{\prime}
\end{gathered}
$$

where $v_{i+1}{ }^{\prime}$ is the updated velocity of $v_{i+1}$ after velocity control, $\alpha$ is the angle between $v_{i+1}$ and the direction from $p_{i+1}$ to gbest and $p_{i+1}{ }^{\prime}$ is the updated local best position of $p_{i+1}$ after velocity control.

At the $i$-th iteration, particle velocity and position are updated by (48) and (49), and then the final particle position and velocity are updated by the velocity control strategy.

The iteration procedure of CCPSO is as follows:

Begin.

Step 1. Input particle position and velocity.

Step 2. Update the particle position and velocity based on (39) and (40).

Step 3. The position of particles is limited in $\left(0, \frac{\pi}{2}\right)$.

Step 4. If $\alpha>0$, turn to Step 5; otherwise, turn to Step 6; $\alpha$ is calculated based on (41).

Step 5. Update particle position and velocity based on (42) and (43).

Step 6. Call fitness solving module.

Step 7. Update the local best and the global best.

Step 8. Turn to Step 1 until all the particles are traversed.

End.

\subsubsection{Particle Chaos Method}

Chaos is a general nonlinear phenomenon; its behavior is complex and similar to random, but it has exquisite internal laws. Because of the ergodicity of chaos, the optimal search using chaotic variables is more advantageous than the random search with blind disorder, and it can avoid the algorithm falling into local optimum. The chaotic mapping method is one of the core contents of chaos search. There are many researches on onedimensional or two-dimensional chaotic mapping [24-27]. These studies mainly aimed at improving the ergodicity of particles in chaotic mapping space and other related features. The studies about chaotic mapping for higher dimensional space are few in number. In general scheduling problems, multiple tasks and scheduling objects are involved, so applying chaotic search methods to scheduling problems requires reconsideration of the applicable chaotic strategies.

Equation (44) is a commonly used one-dimensional chaotic mapping method. This mapping space is simple to use, and the mapped particles have good randomness and convenience.

$$
\operatorname{map}(x)=4 x(1-x), x \in(0,1)
$$

where $\operatorname{map}(x)$ is chaotic mapping function, and $\mathrm{x}$ is the input value.

The particle chaos procedure is as follows:

Begin.

Step 1. Input particle position and velocity.

Step 2. Set $p_{c}$ (the probability of chaos), and generate a random number $x$ in $(0,1)$. If $x<p_{c}$, turn to Step 3; otherwise, turn to Step 4. 
Step 3. Chaos starts from the second element of the particle, and the mapping value is calculated based on the following formula:

$$
P_{i j k}=\min \left(\frac{P_{i j k-1}}{0.5 \pi}+0.01,0.9\right)
$$

where $P_{i j k}$ : The position of the $k$-th element of the $j$-th dimension in the $i$-th particle.

Step 4. Turn to Step 1 until all the particles are traversed.

End.

\subsubsection{The Algorithm Steps}

CCPSO is used to solve the hybrid scheduling for multi-equipment at the U-shape trafficked automated terminal. As mentioned in Section 3, in the hierarchical architecture model, the loop body, the algorithm module and the scheduling module are independent as a whole, and the changes in one module do not affect other parts. Figure 8 shows the loop body (the flowchart of CCPSO) of the hierarchical architecture model in this paper. The whole process is divided into three parts: particle initialization, particle swarm iteration and particle chaos. The input general task information contains the task type and the container locations in the yard. The container location information includes yard, bay and row, which represent the number of the yard block, bay and row locations. Since the hybrid scheduling in this paper involves three kinds of scheduling machines, there are three subgroups in particle swarm.

The iteration procedure of CCPSO for hybrid scheduling optimization of YC, AGV and ET at the U-shape trafficked automatic terminal is as follows:

Begin.

Step 1. Input the general tasks information.

Step 2. Initialize scheduling decision variable YC, AGV and ET.

Step 3. Initialize particle swarm parameters.

Step 4. Call the particle swarm iteration module and input the particle swarm.

Step 5. Call the particle chaos module and input the particle swarm.

Step 6. Turn to Step 1 until the iteration or the computation time reaches the limit value. End.

Figure 9 shows the overall cycle flow of the circulation layer and how each layer relates to the others. Figure 9 summarizes the basic layers of the scheduling problem. They are interconnected through the loop body given by the circulation layer and do not affect each other. This makes the layered structure of this article applicable to different scheduling problems. For example, in other scheduling research, more scheduling equipment needs to be considered, and then it is necessary to build a problem model from scratch and perform algorithm design. The new structure reduces the workload. Under the new structure, only the scheduling module of the scheduling layer needs to be designed at this time.

Section "Dynamic scheduling" has explained the use of dynamic idea to solve the fitness value and container transportation sequence of each equipment. The static solution strategy used in the existing literature cannot be applied to the mixed constraint in the hybrid scheduling problem with multiple equipment types, because, under this type of constraint, the equipment transportation is affected by same type of equipment and different types of equipment. Solving the status of each equipment at each moment is time-consuming and inefficient. When the transportation equipment is doing the task, it is not necessary to pay attention to the status of other equipment at each moment; only when $\mathrm{YC}, \mathrm{AGV}$ or ET arrives at the designated handover location, it needs to access whether the docking equipment is in place. When the AGV or ET is ready to move, it needs to access whether other equipment is on its current lane to prevent collisions. 


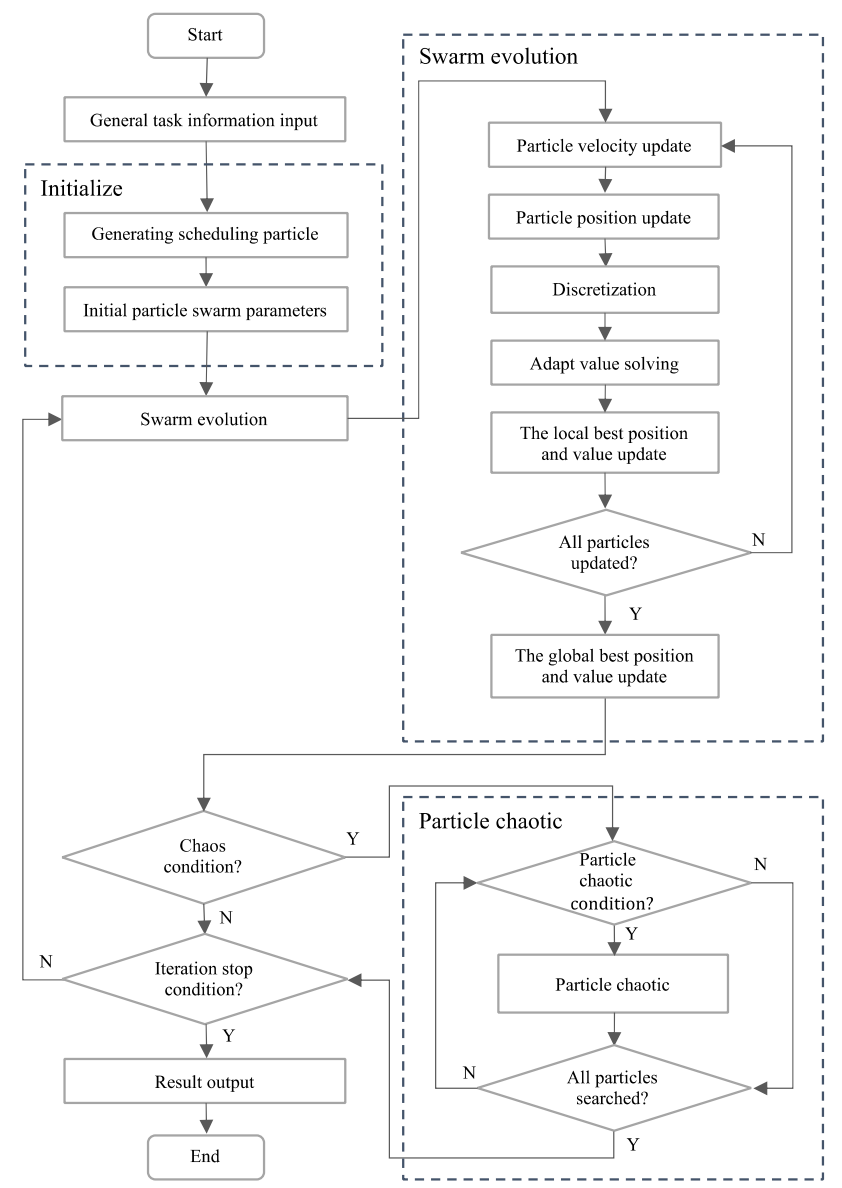

Figure 8. CCPSO flowchart.

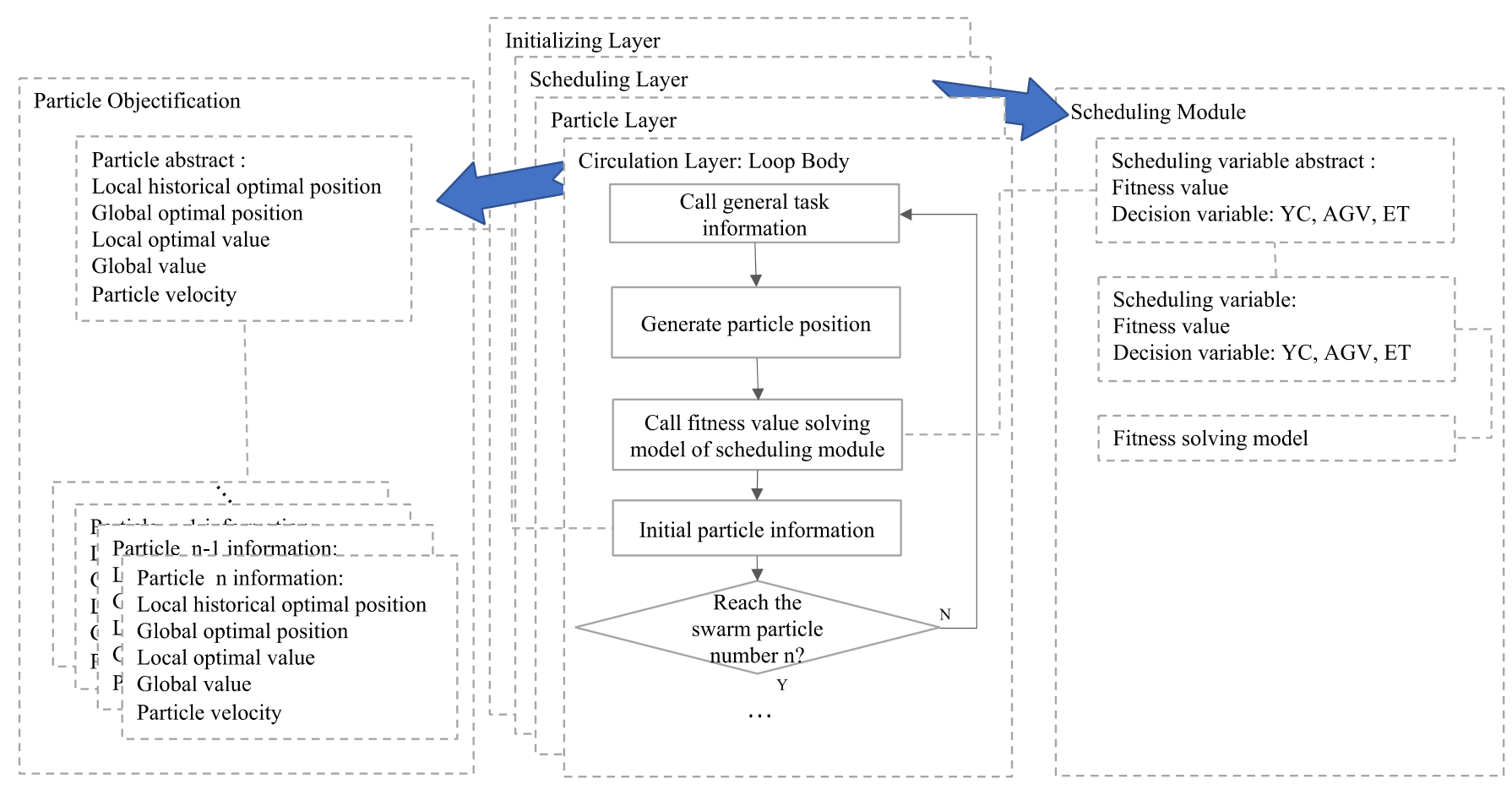

Figure 9. Module layer process. 
The interaction of dynamic information flow was explained in Section "Dynamic scheduling", and Figure 10 shows the solution process for fitness value and equipment task scheduling sequence based on the dynamic scheduling strategy. For a static scheduling result (YC, AGV and ET container transportation sequence), set $\mathrm{YC}$ task pointers for all YCs to traverse. For the task pointed by each YC pointer, the corresponding AGV or ET is queued at the yard block entrance. The sooner the $\mathrm{YC}$ arrives at the designated bay, the sooner the AGV or ET arrives at the entrance, and the earlier the container is transported; the AGV or ET transporting the container is closer to the forefront of the queue. Next, the container at the front of the queue is processed. As shown in Figure 10, the AGV or ET acts by accessing the yard block lane occupation information (waiting to reach the target location). The yard block lane is divided according to the bay position, and the lane occupation information records the latest release time of each lane position, after the release time the lane position can be occupied. When the task pointer traverses all tasks, the solving process ends and outputs the scheduling result, which includes the YC, AGV and ET container transportation sequences; each YC, AGV and ET container task's completion time; and total completion time (fitness value).

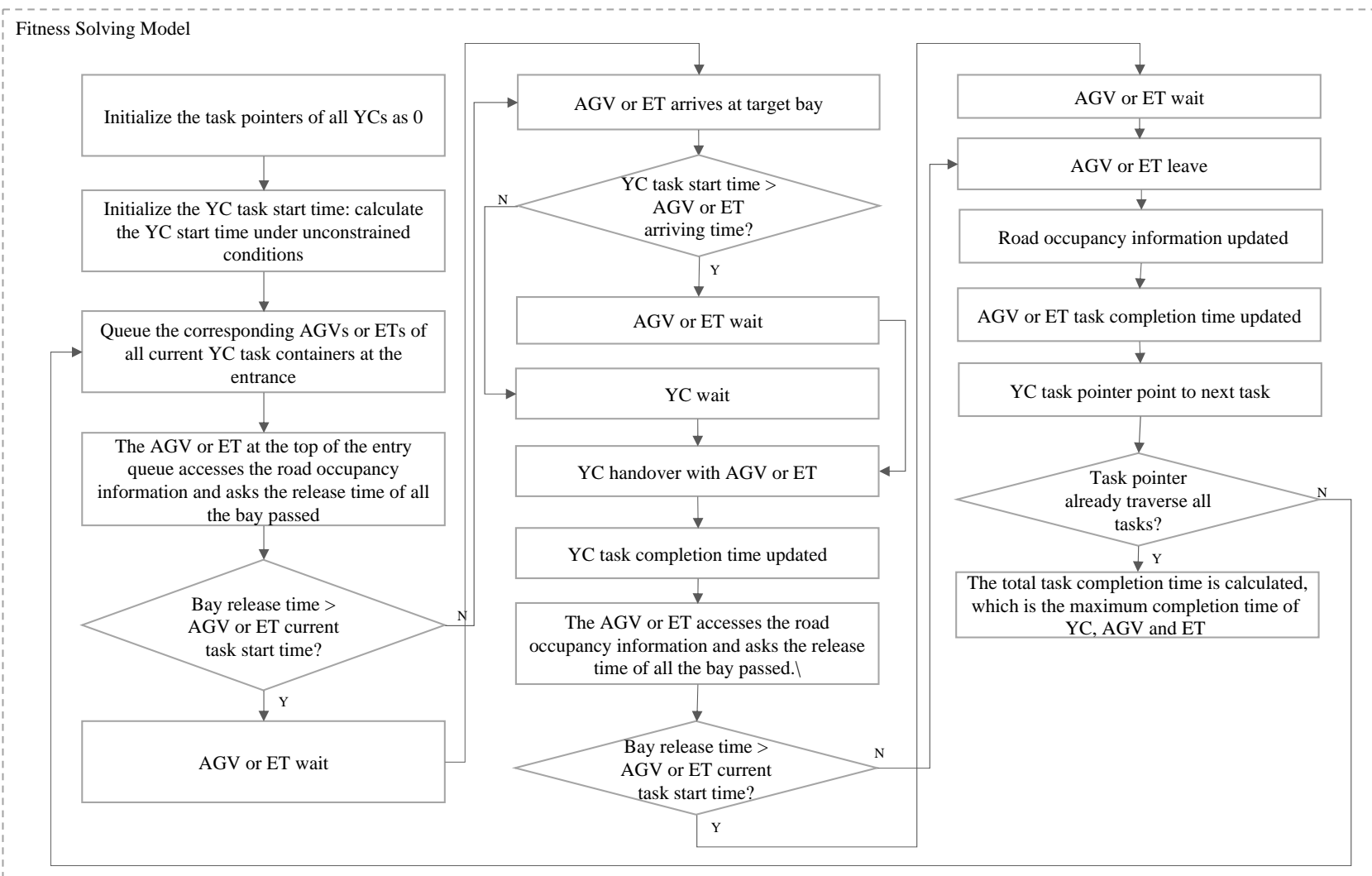

Figure 10. Dynamic solving process.

\section{Validation and Result Analysis}

\subsection{Parameter Settings}

Here, two blocks are considered in the U-shape trafficked automated terminal. The size of each yard is set as bay $\in[0,7]$, row $\in[0,5]$ and fall $\in[0,5]$ (the yard parameters refers to the Qinzhou Port). Two YCs, four AGVs and enough ETs are arranged for each block. In CCPSO, the particle swarm size is 20; the inertia weight $\omega$ is 0.729 ; the cognitive and social coefficients, $c_{1}$ and $c_{2}$, are both 1.494; $r_{1}$ and $r_{2}$ are random numbers in $(0,1)$; and the chaos probability, $p_{c}$, is $30 \%$.

The computer equipped with $2.3 \mathrm{GHz}$ quad-core Intel Core i5, 8 GB $2133 \mathrm{MHz}$ LPDDR3 and Python 3.8 was used in following scheduling experiments. 


\subsection{Numerical Example}

This study is the first to discuss the hybrid scheduling of three types of equipment: AGV, YC and ET at the U-shape trafficked automated terminal. A feasible solution should not only satisfy the path constraint of AGV and ET, but also applies to the coupling relationship among AGV, ET and YC. Table 6 shows the solving speed of feasible solutions under different number of containers and AGVs, where $N_{C}$ denotes the number of containers, $N_{A G V}$ denotes AGV quantity, "Completion Time" denotes the completion time of the transportation and "CPU Time" denotes the calculation time required for the solution, and the result is rounded to two decimal places. It can be seen that the actual calculation time for solving the model in this paper is still controlled within $1 \mathrm{~s}$ after the container reaches 10,000 . Due to the fast solving speed of feasible solutions in this paper, the following mainly verifies the superiority of CCPSO by comparing the optimal solutions obtained by different algorithms in different container numbers, different AGV numbers and different iterations.

Table 6. Solving speed.

\begin{tabular}{cccc}
\hline $\mathbf{n}$ & Size & Completion Time (Seconds) & CPU Time (Seconds) \\
\cline { 2 - 3 } & $N_{C} \times N_{A G V}$ & & 0.00 \\
1 & $20 \times 4$ & 390 & 0.00 \\
2 & $100 \times 8$ & 1948 & 0.25 \\
3 & $200 \times 8$ & 3529 & 0.26 \\
4 & $500 \times 16$ & 9344 & 0.27 \\
5 & $1000 \times 16$ & 18,108 & 0.40 \\
6 & $2000 \times 32$ & 36,624 & 0.55 \\
7 & $10,000 \times 48$ & 185,879 & \\
\hline
\end{tabular}

The feasibility of the model and algorithm were verified in this paper. Appendix A Table A1 contains the task information that YC, AGV and ET need to perform in a smallscale calculation example containing 20 containers, where the number denotes the container number; the type denotes the task type of the current container; the block denotes the yard block number where the container is located; and the Bay, Row and Fall respectively denote the specific three-dimensional coordinate position of the container in the yard. The direction of Bay is parallel to YC carts. Appendix A Table A1 shows the optimal scheduling arrangements of $Y C, A G V$ and ET after the solution is solved within 50 iterations. From the left-most column to the right-most column, the scheduling arrangement is two YCs at the No. 0 yard block, two YCs at No. 1 yard block, 4 AGVs and the queuing sequence of ET transporting corresponding containers at the entrance. Each column of Appendix A Table A2 respectively indicates the order in which YC and AGV transport containers. The ET column indicates the order in which ET transporting the corresponding container enters the yard block, and the scheduling sequence is from top to bottom.

Appendix A Figures A1-A4 show the positions and time relationships of AGV, ET, and $Y C$ at various times. The horizontal axis denotes time, and the vertical axis uses bay bits to denote the current position of each equipment along the track of YC cart. The layout of the yard is shown in Figure 3. The lanes of No. 1 block and No. 2 block are 0-7 and 8-14, respectively. In Appendix A Figure A1, lines with four colors represent four different AGVs, respectively. Since ET does not belong to port internal equipment, the lines representing ET are all red in Appendix A Figure A2. If the line crossing occurs at the same bay at the same time in Appendix A Figures A1 and A2, it means that there is a transport vehicle interference here. The entrance is assumed to be located at the 0 -th bay. Due to the situation where the AGVs queue at the yard entrance, parallel lines appear at the 0 bay position in Appendix A Figure A1, which means that the AGV of the corresponding color is waiting in the queue. There is no line crossing in Appendix A Figures A1 and A2, so it can be verified that the constraint model meets the requirements. Appendix A Figure A4 shows the convergence of the optimal value of the algorithm in iterations. It can be seen that the optimal solution initially obtained is above 320 and then approaches to 285 after 
update iterations. In fact, the final solution result tends to be better as iteration increases, because CCPSO has the characteristic of not falling into local optimum. This characteristic will be further verified in subsequent experiments.

\subsection{Simulation Comparison}

To further verify the performance of CCPSO, different algorithms are calculated for comparison under different number of containers and AGVs. They include the traditional particle swarm optimization algorithm (PSO), the adaptive particle swarm optimization algorithm (APSO) developed by Cong et al. [22] and the random point particle swarm optimization algorithm (RPPSO) developed by Dawei et al. [28]. The particle processing method and fitness value solution models are based on the model in this study. All algorithms are calculated in the same iteration. Since the calculation time required in a single iteration of each algorithm is different, for each algorithm, we take the result at the same time point before the end of the calculation, rather than at the end of the iteration.

The velocity and position iteration method of PSO is based on (40) and (41). The percentage difference between PSO and CCPSO is represented by GAPPSO-CCPSO in (46). A positive result indicates that CCPSO is better, and a negative result indicates that PSO is better.

$$
G_{\text {PAS }-C C P S O}=\frac{T_{P S O}-T_{C C P S O}}{T_{C C P S O}} \times 100(\%)
$$

where $T_{P S O}$ is the completion time of PSO, and $T_{C C P S O}$ is the completion time of CCPSO.

Similar to (46), the calculation formula for percentage deference between APSO, RPPSO and CCPSO is as follows:

$$
\begin{aligned}
G A P_{A P S O}-C C P S O & =\frac{T_{A P S O}-T_{C C P S O}}{T_{C C P S O}} \times 100(\%) \\
G A P_{R P P S O-C C P S O} & =\frac{T_{R P P S O}-T_{C C P S O}}{T_{C C P S O}} \times 100(\%)
\end{aligned}
$$

where $T_{A P S O}$ is the completion time of APSO, and $T_{R P P S O}$ is the completion time of RPPSO.

Appendix A Table A3 gives the final task completion time of PSO, APSO, RPPSO and CCPSO under different container quantity, AGV numbers and iteration. In Appendix A Table A3, each size $\left(N_{c} \times N_{A G V} \times N_{I}\right)$ represents a group of experiments, and each group of experimental data is the average result of three independent experiments. In size $\left(N_{c} \times N_{A G V} \times N_{I}\right), N_{I}$ represents the maximum number of iterations. From Appendix A Table A3, in the comparison simulation, the average gap of completion time can show the ability between CCPSO, PSO, APSO and RPPSO. It can find that CCSPO can save yard operation time about $1.162 \%, 1.418 \%$ and $1.695 \%$ more than others. Therefore, the searching ability of CCPSO is indeed better than other algorithms.

Figure 11 shows the comparison of the solution obtained by four algorithms under the same number of containers $(\mathrm{N})$, different AGV number and maximum number of iteration. In each subgraph, the solid and dashed lines correspond to different AGV quantity, respectively. Different colors are used to indicate different algorithms respectively, and the corresponding relationship is as marked. Gray, orange, green and blue indicate PSO, APSO, RPPSO and CCPSO, respectively. It can be seen that the completion times obtained by CCPSO are basically below other lines in each subgraph, thus indicating that CCPSO's solution results are better in most cases. When the number of containers increases, the solution of other algorithms gradually becomes flat, while the solution of CCPSO still maintains a downward trend, which means that CCPSO is more adaptable in complex situations and different allowed solution times. The experimental results show that CCPSO does not only find a near optimal solution in a short time, but also has a better ability to perform a global search. 

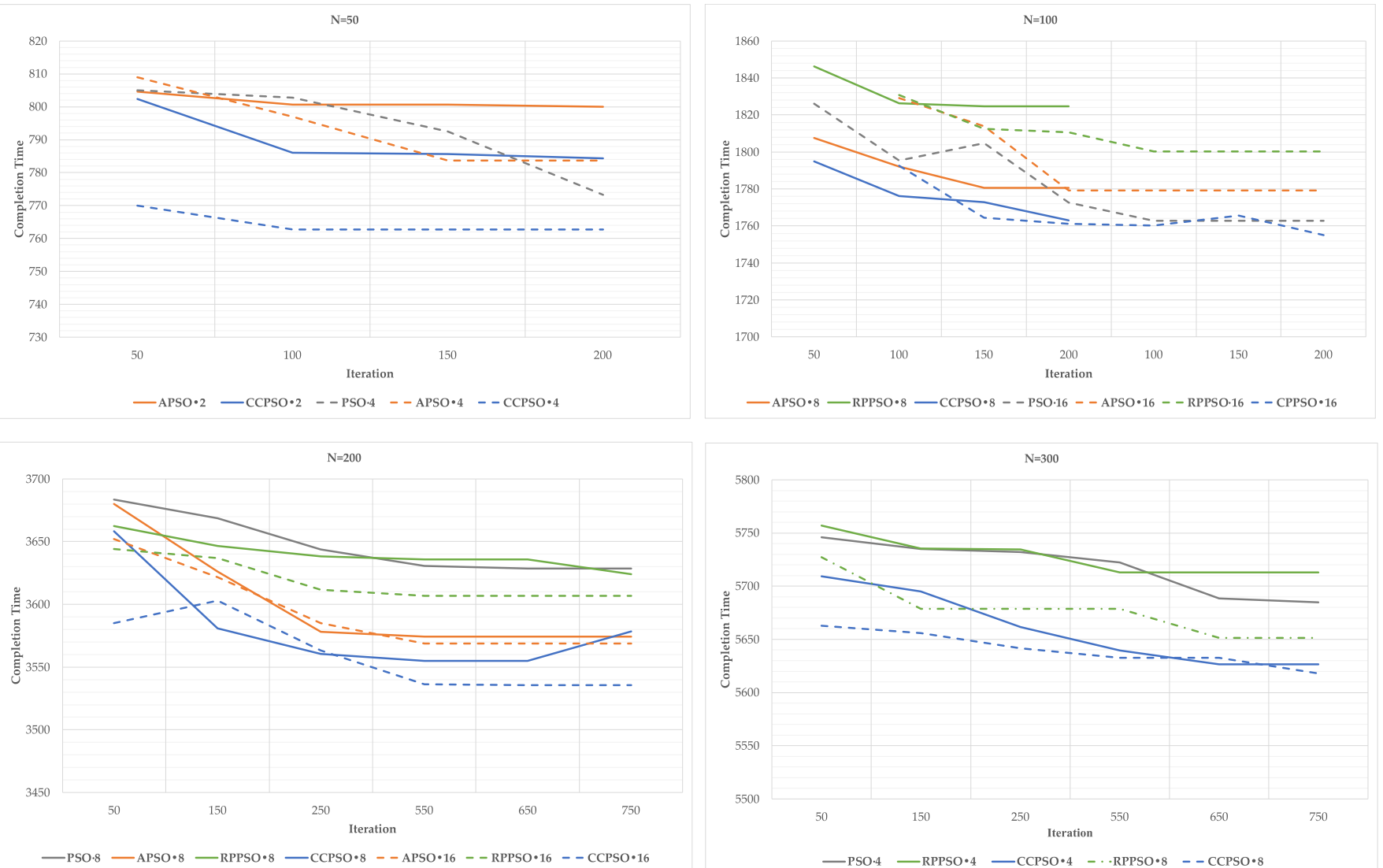

Figure 11. Comparison chart for PSO, APSO, RPPSO and CCPSO.

\subsection{Sensitivity Analysis}

The AGV quantity $\left(N_{A G V}\right)$ and the iteration number $\left(N_{I}\right)$ are considered separately as the sensitive sources, and the fitness values (completion times) of comparison algorithms are output.

In the sensitivity test of AGV quantity allocation, the total number of tasks is set to 100 , the iteration number is set to 50 and the AGV allocation quantity 8 's change rate is set to $0 \%$. The AGV detection rate (AGV quantity's change rate) is $-75 \%,-50 \%,-25 \%, 0 \%$, $25 \%, 50 \%$ and $75 \%$. The data obtained are shown in Table 7 . Figure 12 shows the sensitivity of each algorithm when detecting results in different configurations of AGVs. It can be seen from Figure 12 that PSO's results are sensitive to changes in the number of AGV. When the number of AGV quantity is small, the PSO's results obtained are poor. When the AGV number increases, the PSO's solution results are better than those of other algorithms. On the whole, the solution results of CCPSO are relatively stable, and at the same time, they are relatively close to the optimal results in the control group.

Table 7. Sensitivity test data of AGV quantity allocation.

\begin{tabular}{cccccc}
\hline Size & \multirow{2}{*}{ Detection Rate $\left(\boldsymbol{N}_{A G V}\right)$} & \multicolumn{3}{c}{ Completion Time (Unit Time) } \\
\cline { 3 - 5 }$N_{\boldsymbol{c}} \times \boldsymbol{N}_{A G V} \times \boldsymbol{N}_{\boldsymbol{I}}$ & & PSO & APSO & RPPSO & CCPSO \\
\hline $100 \times 2 \times 50$ & $-75 \%$ & 1889 & 1838 & 1886 & 1851 \\
$100 \times 4 \times 50$ & $-50 \%$ & 1864 & 1840 & 1844 & 1832 \\
$100 \times 6 \times 50$ & $-25 \%$ & 1855 & 1832 & 1842 & 1828 \\
$100 \times 8 \times 50$ & $0 \%$ & 1828 & 1836 & 1826 & 1832 \\
$100 \times 10 \times 50$ & $25 \%$ & 1817 & 1824 & 1842 & 1840 \\
$100 \times 12 \times 50$ & $50 \%$ & 1819 & 1828 & 1840 & 1835 \\
$100 \times 16 \times 50$ & $75 \%$ & 1798 & 1837 & 1821 & 1824 \\
\hline
\end{tabular}




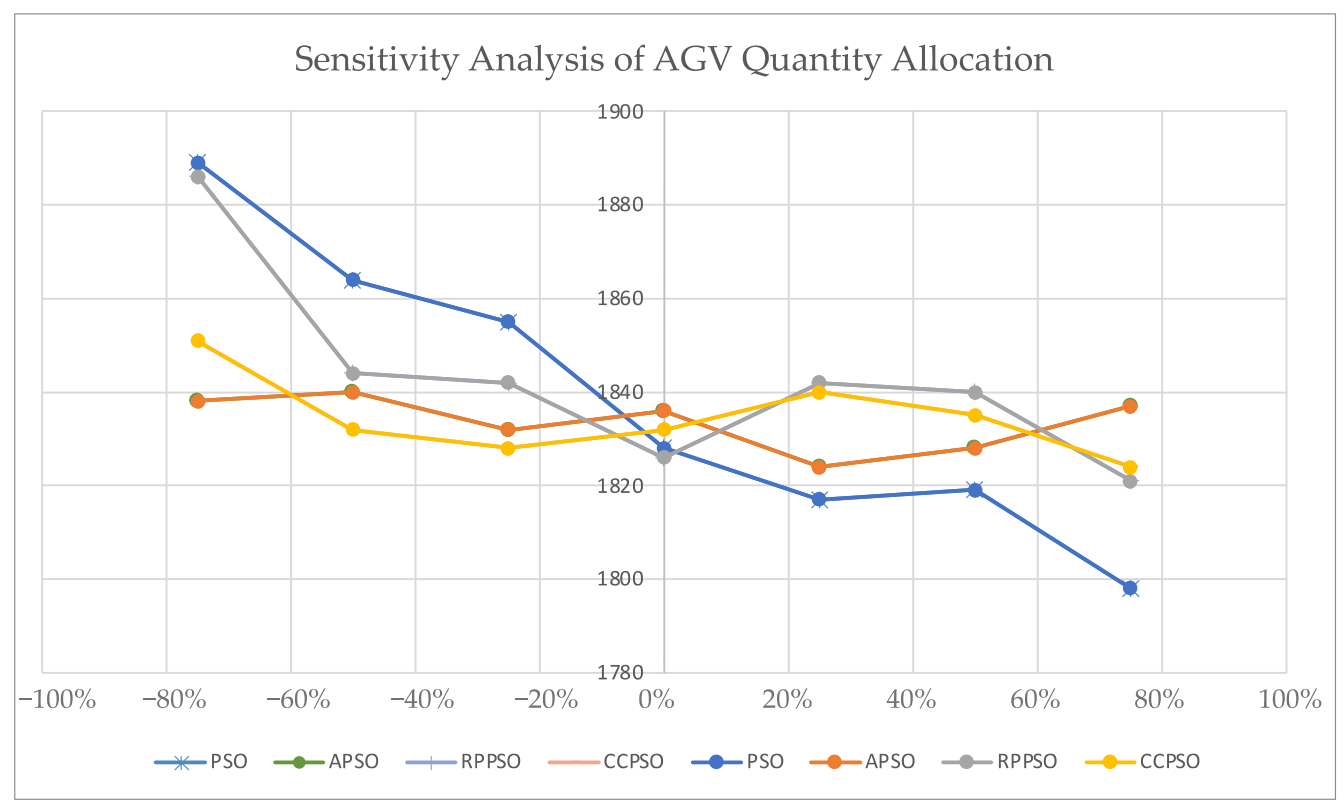

Figure 12. Sensitivity analysis of AGV quantity allocation.

In the sensitivity test of the iteration number, the total number of tasks is set to 100 , the AGV allocation quantity is set to 16 and the iteration number $200^{\prime} s$ change rate is set to $0 \%$. The iteration number's detection rate (iteration number's change rate) is $-75 \%$, $-50 \%,-25 \%, 0 \%, 25 \%, 50 \%$ and $75 \%$. The results obtained are shown in Table 8 . As shown in Figure 13, CCPSO is most sensitive to the iteration number. When the iteration number increases, the result of CCPSO is better, while other algorithms do not appear to be insensitive. When the number of iterations increases, there are no further optimizations of these algorithms.

Table 8. Sensitivity test data of iteration number.

\begin{tabular}{cccccc}
\hline Size & \multirow{2}{*}{ Detection Rate $\left(\boldsymbol{N}_{A G V}\right)$} & \multicolumn{3}{c}{ Completion Time (Unit Time) } \\
\cline { 3 - 5 } $\boldsymbol{N}_{\boldsymbol{c}} \times \boldsymbol{N}_{A G V} \times \boldsymbol{N}_{\boldsymbol{I}}$ & & PSO & APSO & RPPSO & CCPSO \\
\hline $100 \times 16 \times 50$ & $-75 \%$ & 1798 & 1837 & 1821 & 1824 \\
$100 \times 16 \times 100$ & $-50 \%$ & 1798 & 1812 & 1821 & 1824 \\
$100 \times 16 \times 150$ & $-25 \%$ & 1798 & 1807 & 1821 & 1822 \\
$100 \times 16 \times 200$ & 0 & 1798 & 1801 & 1821 & 1787 \\
$100 \times 16 \times 250$ & $25 \%$ & 1798 & 1801 & 1821 & 1782 \\
$100 \times 16 \times 300$ & $50 \%$ & 1798 & 1801 & 1821 & 1779 \\
$100 \times 16 \times 350$ & $75 \%$ & 1798 & 1801 & 1821 & 1779 \\
\hline
\end{tabular}

In summary, CCPSO has better adaptability in practical applications: when the allocation quantity of AGV is small, it can obtain a near-optimal solution in a shorter time; at the same time, CCPSO is sensitive to the iteration number. It can be seen that CCPSO has a better ability to search solutions and can adapt to different resource allocation and production requirements (it takes a shorter time to solve the near-optimal solution, or finds a better solution when the solution time is sufficient). 


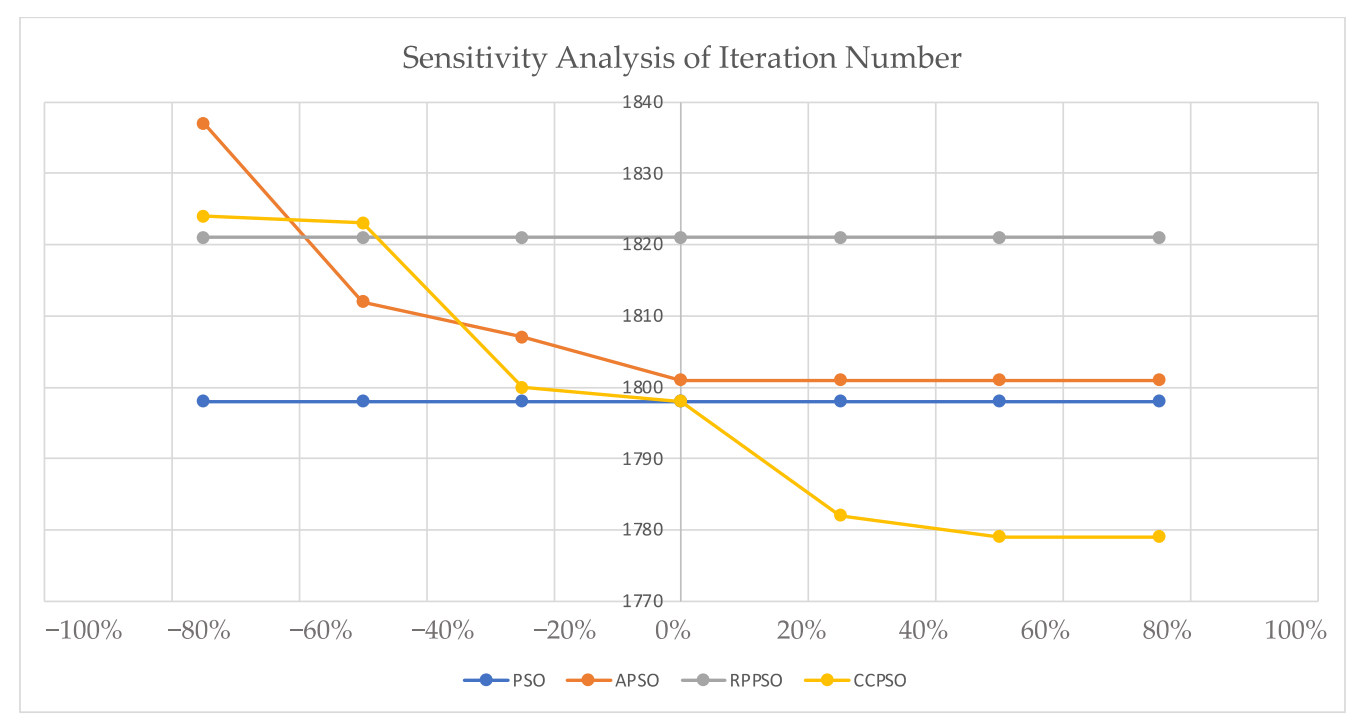

Figure 13. Sensitivity analysis of iteration number.

\section{Conclusions}

ZPMC proposed the U-shape trafficked terminal to improve the terminal efficiency. Meanwhile, it brought a new problem: mixed constraints between YC, AGV and ET in yard scheduling. A hybrid scheduling problem of YC AGV and ET at the U-shape trafficked terminal was studied. First, the scheduling objects involved in multi-equipment scheduling, such as YC, AGV and ET, were abstracted and formed into modules. Then each module was divided into four levels, according to its internal logical relationship: initialization layer, loop layer, particle module layer and scheduling module layers. Finally, a multiequipment scheduling layered model architecture was established. The architecture, which has good scalability, is suitable for various multi-equipment hybrid scheduling problems. It can effectively avoid the duplication of work and improve research efficiency. A static and dynamic mixed scheduling method was put forward to solve the fitness value, which is suitable for the multi-equipment mixed constraint problem. The mapping space of decision variables to particles was optimized, and the CCPSO algorithm with particle chaos strategy and particle iterative speed control strategy was presented for the hybrid scheduling problem. The simulation experiment verifies the speed and effectiveness of the algorithm by using PSO, APSO, RPPSO and CCPSO for comparison. The comparative simulations are carried out under a different number of containers, different number of AGV and different maximum number of iterations. As the simulation showed, the CCSPO saves yard operation time about $1.162 \%, 1.418 \%$ and $1.695 \%$ better than PSO, APSO and RPPSO respectively, on average. The sensitivity test of AGV quantity and iteration number further shared the performance of CCPSO. The results show that, when the number of the container and AGV increases, the advantages of CCPSO are more obvious.

In the current work, we only considered the scheduling of one type of yard lane layout. In the future, the layout of multiple yard lanes will be considered. The scheduling of QCs will be considered, together with those of YCs, AGVs and ETs. This will involve more mixed constraints, such as AGV constraints from QCs to yards, YC non-crossing constraints, ET delays, etc. In addition, considering the inevitable uncertainty in terminal operations, the robust optimization [19] will also be considered.

Author Contributions: Conceptualization, resources, review and editing, J.L.; methodology, software, validation, formal analysis, writing, J.Y.; supervision, funding acquisition, B.X.; project administration, Y.Y.; resources, F.W. and H.S. All authors have read and agreed to the published version of the manuscript. 
Funding: This research was funded by the National Natural Science Foundation Project of China, grant number 52102466 and the Natural Science Foundation Project of Shanghai, grant number 21ZR1426900.

Data Availability Statement: The data used to support the findings of this study are included within the article.

Conflicts of Interest: The authors declare that there is no conflict of interest regarding the publication of this paper.

\section{Appendix A}

Table A1. Numerical example: general tasks information.

\begin{tabular}{cccccc}
\hline $\mathbf{N}_{\mathbf{C}}$ & Type & Block & Bay & Row & Fall \\
\hline 1 & 4 & 1 & 4 & 2 & 5 \\
2 & 1 & 0 & 3 & 1 & 5 \\
3 & 4 & 1 & 1 & 2 & 5 \\
4 & 1 & 1 & 4 & 2 & 2 \\
5 & 2 & 0 & 7 & 2 & 3 \\
6 & 1 & 0 & 6 & 2 & 1 \\
7 & 3 & 1 & 6 & 2 & 5 \\
8 & 4 & 1 & 1 & 2 & 2 \\
9 & 1 & 0 & 1 & 2 & 1 \\
10 & 2 & 0 & 1 & 3 & 1 \\
11 & 3 & 1 & 4 & 4 & 5 \\
12 & 3 & 1 & 2 & 1 & 5 \\
13 & 2 & 0 & 2 & 2 & 3 \\
14 & 1 & 1 & 5 & 2 & 1 \\
15 & 3 & 1 & 5 & 2 & 4 \\
16 & 2 & 1 & 2 & 2 & 4 \\
17 & 3 & 1 & 5 & 3 & 3 \\
18 & 3 & 1 & 5 & 4 & 5 \\
19 & 3 & 1 & 0 & 2 & \\
20 & 3 & 1 & 7 &
\end{tabular}

Table A2. Numerical experiment: allocation result of each equipment.

\begin{tabular}{|c|c|c|c|c|c|c|c|c|}
\hline YC0 & YC1 & YC2 & YC3 & AGV0 & AGV1 & AGV2 & AGV3 & ET \\
\hline 5 & 2 & 16 & 19 & 4 & 14 & 5 & 9 & 11 \\
\hline 6 & 9 & 12 & 8 & 10 & 13 & 16 & 6 & 19 \\
\hline \multirow[t]{9}{*}{13} & 10 & 3 & 15 & 2 & & & & 20 \\
\hline & & 17 & 1 & & & & & 18 \\
\hline & & 18 & 4 & & & & & 7 \\
\hline & & 14 & 11 & & & & & 12 \\
\hline & & 20 & 7 & & & & & 3 \\
\hline & & & & & & & & 8 \\
\hline & & & & & & & & 1 \\
\hline & & & & & & & & 15 \\
\hline & & & & & & & & 17 \\
\hline
\end{tabular}




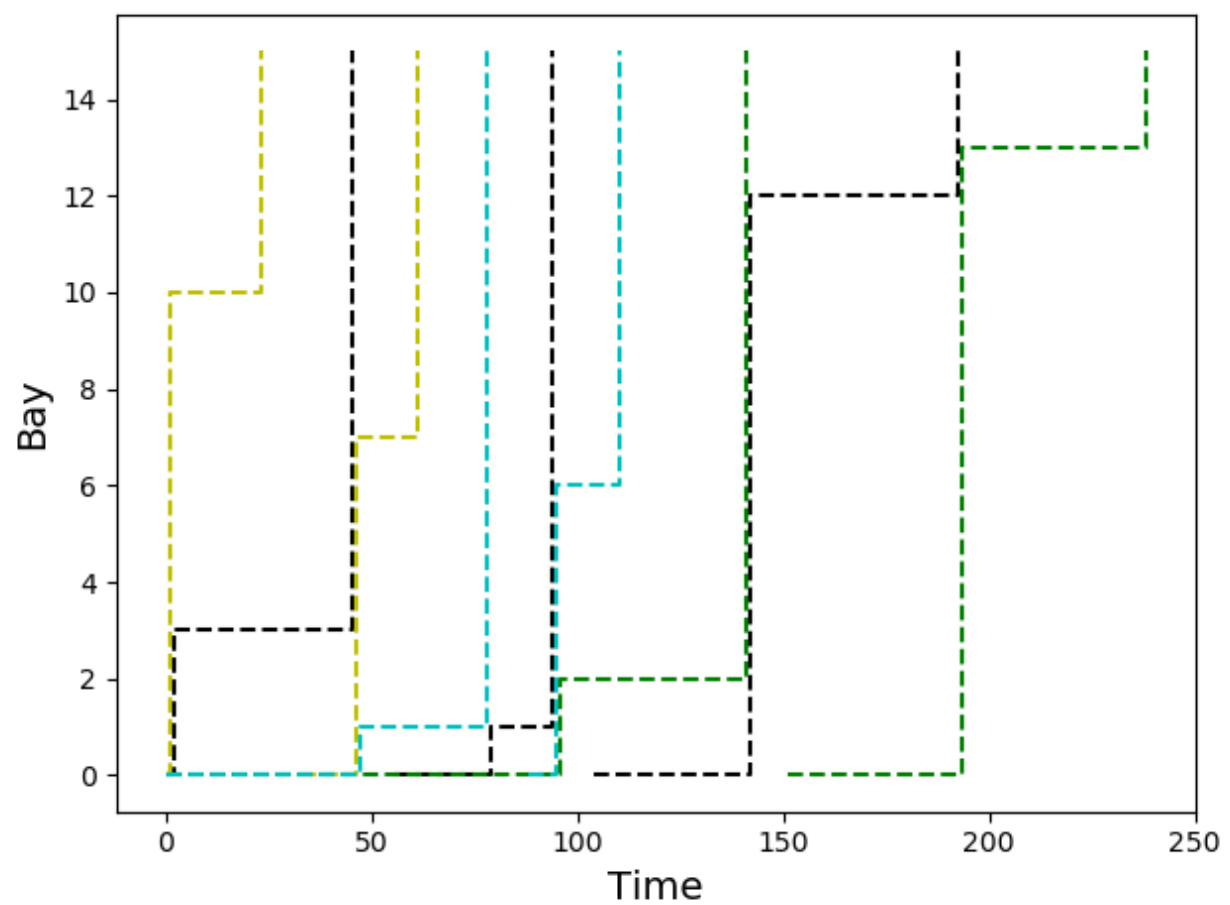

Figure A1. Numerical experiment: AGV time-space diagram.

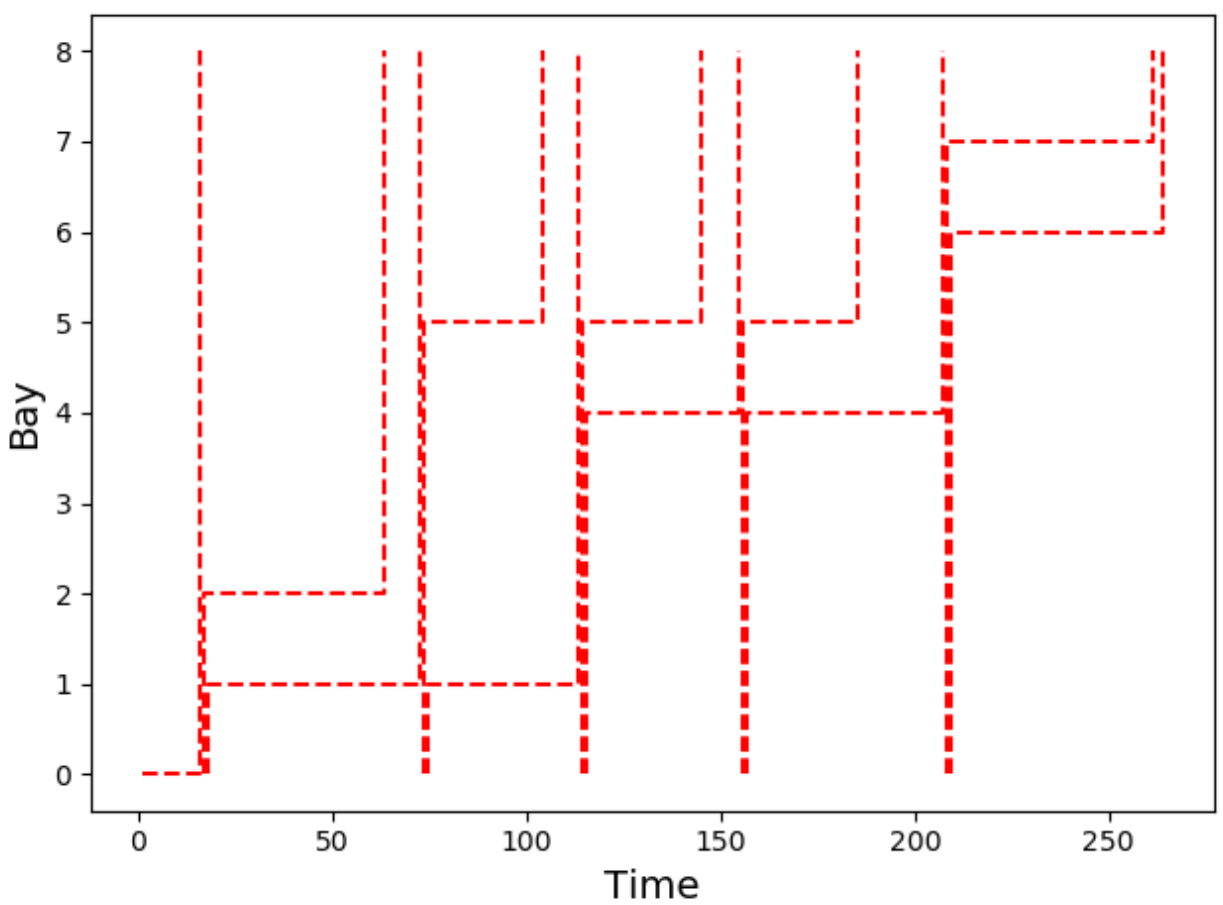

Figure A2. Numerical experiment: ET time-space diagram. 


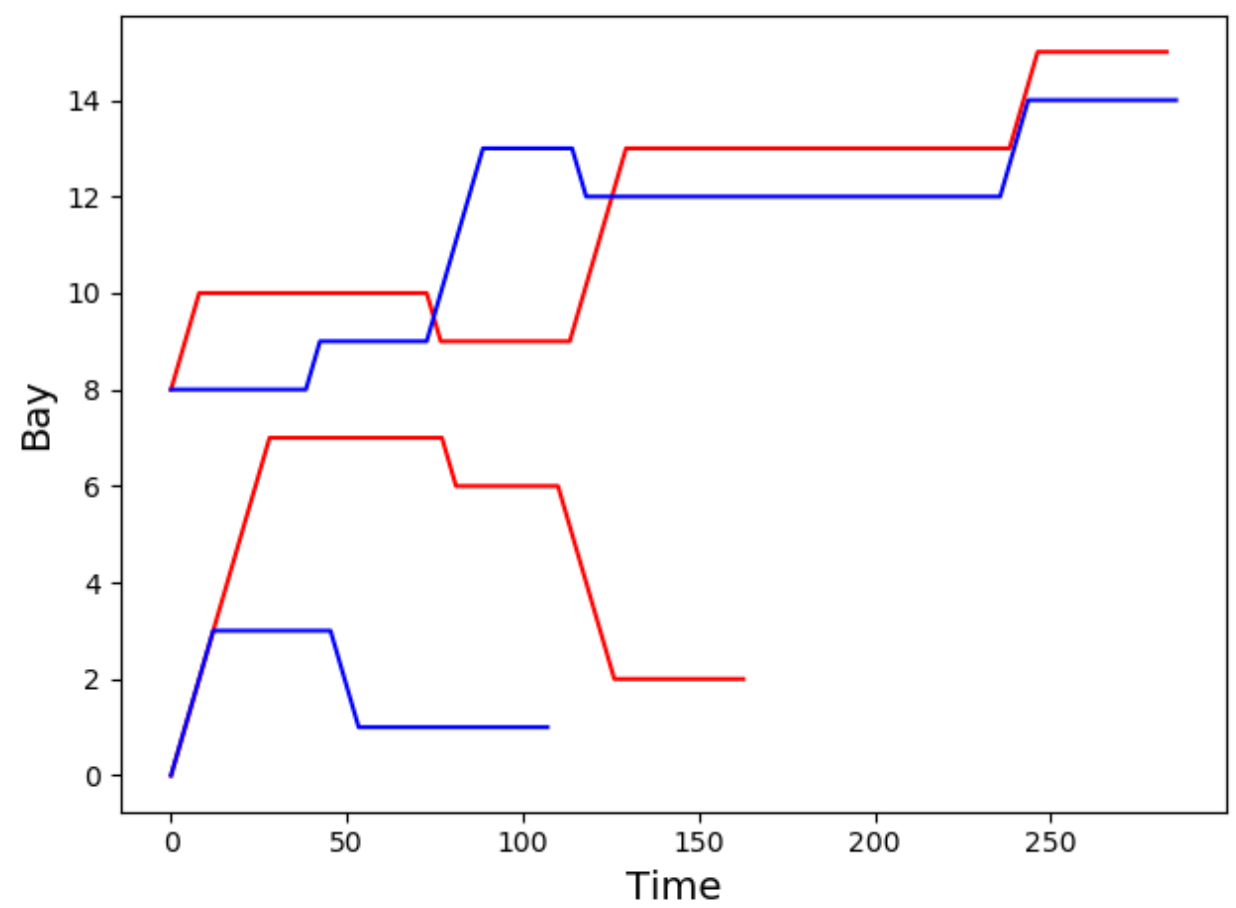

Figure A3. Numerical experiment: YC time-space diagram.

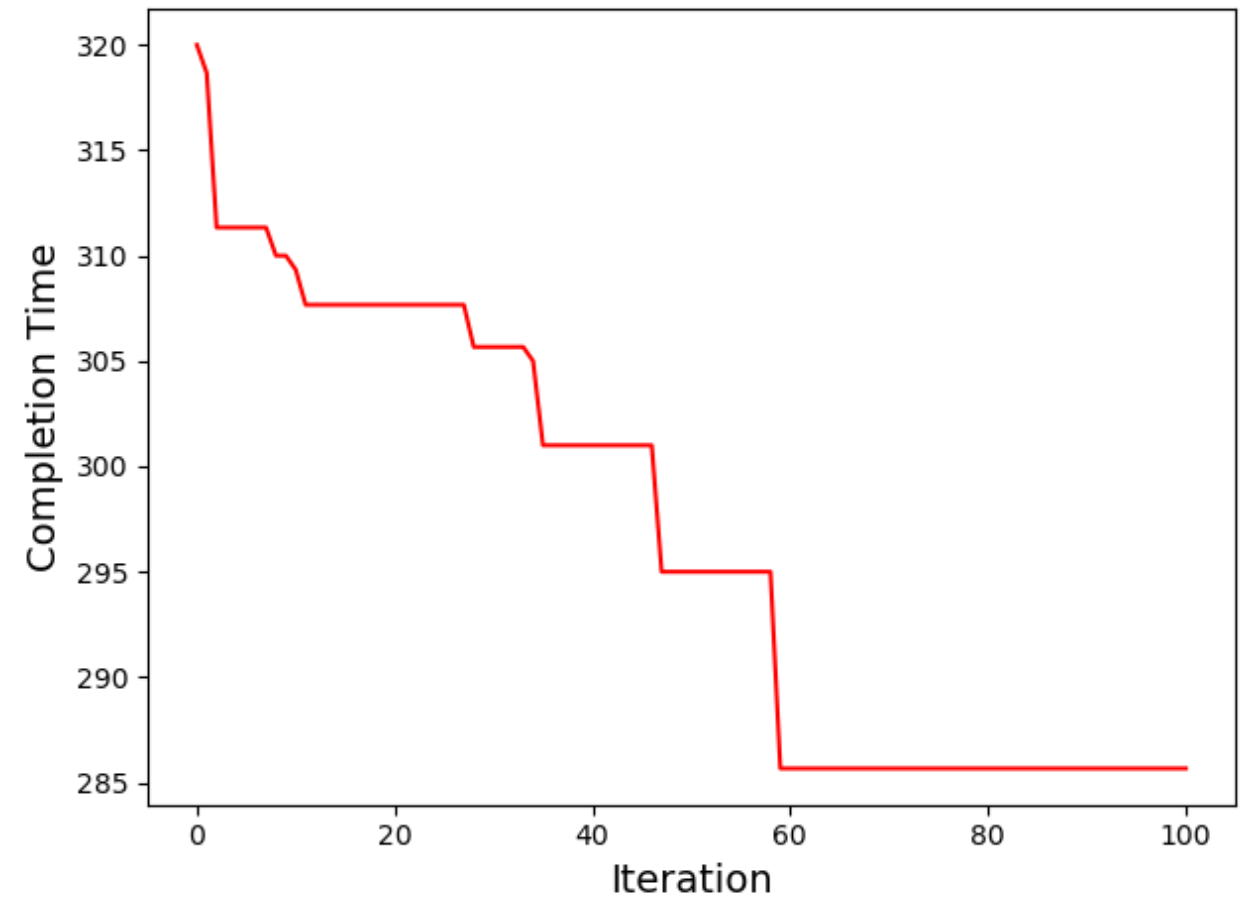

Figure A4. Numerical experiment: optimal solution update.

Table A3. Comparison experiment of PSO, APSO, RPPSO and CCPSO.

\begin{tabular}{ccccccccc}
\hline $\mathbf{n}$ & Size & \multicolumn{3}{c}{ Completion Time (Unit Time) } & & \multicolumn{2}{c}{ GAP (\%) } \\
\cline { 2 - 6 } & $N_{c} \times N_{A G V} \times N_{I}$ & PSO & APSO & RPPSO & CCPSO & PSO & APSO & RPPSO \\
\hline 1 & $50 \times 2 \times 50$ & - & 805 & - & 802 & - & 0.374 \\
2 & $50 \times 2 \times 100$ & - & 801 & - & 786 & - & 1.908 & - \\
3 & $50 \times 2 \times 150$ & - & 801 & - & 786 & - & 1.908 & - \\
4 & $50 \times 2 \times 200$ & - & 800 & - & 784 & - & 2.041 & - \\
\hline
\end{tabular}


Table A3. Cont.

\begin{tabular}{|c|c|c|c|c|c|c|c|c|}
\hline \multirow[t]{2}{*}{$\mathbf{n}$} & \multirow{2}{*}{$\frac{\text { Size }}{N_{c} \times N_{A G V} \times N_{I}}$} & \multicolumn{4}{|c|}{ Completion Time (Unit Time) } & \multicolumn{3}{|c|}{ GAP (\%) } \\
\hline & & PSO & APSO & RPPSO & CCPSO & PSO & APSO & RPPSO \\
\hline 5 & $50 \times 4 \times 50$ & 805 & 809 & - & 770 & - & 5.065 & - \\
\hline 6 & $50 \times 4 \times 100$ & 803 & 797 & - & 763 & - & 4.456 & - \\
\hline 7 & $50 \times 4 \times 150$ & 793 & 784 & - & 763 & - & 2.752 & - \\
\hline 8 & $50 \times 4 \times 200$ & 773 & 784 & - & 763 & - & 2.752 & - \\
\hline 9 & $100 \times 8 \times 50$ & - & 1808 & 1846 & 1795 & - & 0.724 & 2.841 \\
\hline 10 & $100 \times 8 \times 100$ & - & 1792 & 1826 & 1776 & - & 0.901 & 2.815 \\
\hline 11 & $100 \times 8 \times 150$ & - & 1781 & 1825 & 1773 & - & 0.451 & 2.933 \\
\hline 12 & $100 \times 8 \times 200$ & - & 1781 & 1825 & 1763 & - & 1.021 & 3.517 \\
\hline 13 & $100 \times 16 \times 100$ & 1795 & 1829 & 1831 & 1793 & 0.112 & 2.008 & 2.119 \\
\hline 14 & $100 \times 16 \times 150$ & 1805 & 1814 & 1812 & 1764 & 2.324 & 2.834 & 2.721 \\
\hline 15 & $100 \times 16 \times 200$ & 1773 & 1779 & 1811 & 1761 & 0.681 & 1.022 & 2.839 \\
\hline 16 & $100 \times 16 \times 250$ & 1763 & 1779 & 1800 & 1760 & 0.170 & 1.080 & 2.273 \\
\hline 17 & $100 \times 16 \times 300$ & 1763 & 1779 & 1800 & 1766 & -0.170 & 0.736 & 1.925 \\
\hline 18 & $100 \times 16 \times 350$ & 1763 & 1779 & 1800 & 1755 & 0.456 & 1.368 & 2.564 \\
\hline 19 & $200 \times 8 \times 50$ & 3683 & 3680 & 3662 & 3658 & 0.683 & 0.601 & 0.109 \\
\hline 20 & $200 \times 8 \times 150$ & 3669 & 3626 & 3646 & 3581 & 2.457 & 1.257 & 1.815 \\
\hline 21 & $200 \times 8 \times 250$ & 3644 & 3578 & 3638 & 3560 & 2.360 & 0.506 & 2.191 \\
\hline 22 & $200 \times 8 \times 550$ & 3631 & 3574 & 3636 & 3555 & 2.138 & 0.534 & 2.278 \\
\hline 23 & $200 \times 8 \times 650$ & 3629 & 3574 & 3636 & 3555 & 2.082 & 0.534 & 2.278 \\
\hline 24 & $200 \times 8 \times 750$ & 3629 & 3574 & 3624 & 3578 & 1.425 & -0.112 & 1.286 \\
\hline 25 & $200 \times 16 \times 50$ & - & 3652 & 3644 & 3585 & - & 1.869 & 1.646 \\
\hline 26 & $200 \times 16 \times 150$ & - & 3622 & 3637 & 3603 & - & 0.527 & 0.944 \\
\hline 27 & $200 \times 16 \times 250$ & - & 3585 & 3612 & 3563 & - & 0.617 & 1.375 \\
\hline 28 & $200 \times 16 \times 550$ & - & 3569 & 3607 & 3536 & - & 0.933 & 2.008 \\
\hline 29 & $200 \times 16 \times 650$ & - & 3569 & 3607 & 3536 & - & 0.933 & 2.008 \\
\hline 30 & $200 \times 16 \times 750$ & - & 3569 & 3607 & 3536 & - & 0.933 & 2.008 \\
\hline 31 & $300 \times 4 \times 50$ & 5746 & - & 5757 & 5709 & 0.648 & - & 0.841 \\
\hline 32 & $300 \times 4 \times 150$ & 5735 & - & 5736 & 5695 & 0.702 & - & 0.720 \\
\hline 33 & $300 \times 4 \times 250$ & 5732 & - & 5735 & 5662 & 1.236 & - & 1.289 \\
\hline 34 & $300 \times 4 \times 550$ & 5722 & - & 5713 & 5639 & 1.472 & - & 1.312 \\
\hline 35 & $300 \times 4 \times 650$ & 5689 & - & 5713 & 5627 & 1.102 & - & 1.528 \\
\hline 36 & $300 \times 4 \times 750$ & 5685 & - & 5713 & 5627 & 1.031 & - & 1.528 \\
\hline 37 & $300 \times 8 \times 50$ & - & - & 5727 & 5663 & - & - & 1.130 \\
\hline 38 & $300 \times 8 \times 150$ & - & - & 5679 & 5656 & - & - & 0.407 \\
\hline 39 & $300 \times 8 \times 250$ & - & - & 5679 & 5642 & - & - & 0.656 \\
\hline 40 & $300 \times 8 \times 550$ & - & - & 5679 & 5633 & - & - & 0.817 \\
\hline 41 & $300 \times 8 \times 650$ & - & - & 5651 & 5633 & - & - & 0.320 \\
\hline 42 & $300 \times 8 \times 750$ & - & - & 5651 & 5618 & - & - & 0.587 \\
\hline Average & & & & & & 1.162 & 1.418 & 1.695 \\
\hline
\end{tabular}

\section{References}

1. Sun, Z.W. The world's First! Zhenhua Heavy Industry Releases New Technology for Container Terminal Loading and Unloading, China Water Transport Network 2019. Available online: http://app.zgsyb.com/news.html?aid=530549 (accessed on 1 October 2021).

2. Beibu Gulf Port. Qinzhou Port Automated Container Terminal Completed Renovation. 2021. Available online: https://www. bbwport.cn/a/xinwenzixun/gongsixinwen/938.html (accessed on 1 October 2021).

3. Bowei, X.; Depei, J.; Junjun, L.; Yongsheng, Y.; Furong, W.; Haitao, S. A hybrid dynamic method for conflict-free integrated scheduling optimization in U-shaped automated container terminals. Comput. Ind. Eng. 2021, 162, 107695. [CrossRef]

4. Iris, C.; Lam, J.S.L. A review of energy efficiency in ports: Operational strategies, technologies and energy management systems. Renew. Sustain. Energy Rev. 2019, 112, 170-182. [CrossRef]

5. Bish, E.K.; Leong, T.Y.; Li, C.L.; Ng, J.W.C.; Simchi-Levi, D. Analysis of a new vehicle scheduling and location problem. John Wiley Sons Ltd. 2001, 48, 363-385. [CrossRef]

6. Bish, E.K. A multiple-crane-constrained scheduling problem in a container terminal. Eur. J. Oper. Res. 2003, 144, 83-107. [CrossRef]

7. Heuermann, A.; Duin, H.; Gorldt, C.; Thoben, K.-D. A Concept for Predictability and Adaptability in Maritime Container Supply Chains. In Dynamics in Logistics, LDIC 2018; Freitag, M., Kotzab, H., Pannek, J., Eds.; Springer: Cham, Switzerland, 2018. [CrossRef] 
8. Boysen, N.; Briskorn, D.; Meisel, F. A generalized classification scheme for crane scheduling with interference. Eur. J. Oper. Res. 2017, 258, 343-357. [CrossRef]

9. Ehleiter, A.; Jaehn, F. Scheduling crossover cranes at container terminals during seaside peak times. J. Heuristics 2018, 24, 899-932. [CrossRef]

10. Eilken, A. A decomposition-based approach to the scheduling of identical automated yard cranes at container terminals. J. Sched. 2019, 22, 517-541. [CrossRef]

11. Briskorn, D.; Zey, L. Interference aware scheduling of triple-crossover-cranes. J. Sched. 2020, 23, 465-485. [CrossRef]

12. Zheng, F.; Man, X.; Chu, F.; Liu, M.; Chu, C. Two Yard Crane Scheduling With Dynamic Processing Time and Interference. IEEE Trans. Intell. Transp. Syst. 2018, 19, 3775-3784. [CrossRef]

13. Dik, G.; Kozan, E. A flexible crane scheduling methodology for container terminals. Flex. Serv. Manuf. J. 2017, 29, 64-96. [CrossRef]

14. Zhong, M.; Yang, Y.; Dessouky, Y.; Postolache, O. Multi-AGV scheduling for conflict-free path planning in automated container terminals. Comput. Ind. Eng. 2020, 142, 106371. [CrossRef]

15. Hu, H.; Jia, X.; He, Q.; Fu, S.; Liu, K. Deep reinforcement learning based AGVs real-time scheduling with mixed rule for flexible shop floor in industry 4.0. Comput. Ind. Eng. 2020, 149, 106749. [CrossRef]

16. Iris, C.; Christensen, J.; Pacino, D.; Ropke, S. Flexible ship loading problem with transfer vehicle assignment and scheduling. Transp. Res. Part B-Methodol. 2018, 111, 113-134. [CrossRef]

17. Chen, X.; He, S.; Zhang, Y.; Tong, L.; Shang, P.; Zhou, X. Yard crane and AGV scheduling in automated container terminal: A multi-robot task allocation framework. Transp. Res. Part C 2020, 114, 241-271. [CrossRef]

18. Houming, F.; Zhenfeng, G.; Lijun, Y.; Mengzhi, M. Combined configuration and scheduling optimization of dual trolley quayside crane and AGV in container terminal considering energy saving. Acta Autom. Sin. 2021, 45, 1-16.

19. Iris, C.; Lam, J.S.L. Recoverable robustness in weekly berth and quay crane planning. Transp. Res. Part B-Methodol. 2019, 122, 365-389. [CrossRef]

20. He, J.; Huang, Y.; Yan, W.; Wang, S. Integrated internal truck, yard crane and quay crane scheduling in a container terminal considering energy consumption. Expert Syst. Appl. 2015, 42, 2464-2487. [CrossRef]

21. Shyalika, C.; Silva, T.; Karunananda, A. Reinforcement Learning in Dynamic Task Scheduling: A Review. SN Comput. Sci. 2020, 1, 306. [CrossRef]

22. Cong, H.D.; Hop, V.N.; Anh, T.T.M. Adaptive particle swarm optimization for integrated quay crane and yard truck scheduling problem. Comput. Ind. Eng. 2020, 153, 107075.

23. Tang, L.X.; Zhao, J.; Liu, J.Y. Modeling and solution of the joint quay crane and truck scheduling problem. Eur. J. Oper. Res. 2014, 236, 978-990. [CrossRef]

24. Wei, J.; Li, Z.; You, L.; Guo, Y.; Tang, Z.; Hu, Z. Consider elite chaotic search strategy of indoor pedestrian evacuation model. J. Syst. Simul. 2021, 33, 1609-1616.

25. Yang, K.; Nomura, H. Quantum-Behaved Particle Swarm Optimization with Chaotic Search. IEICE Trans. Inf. Syst. 2008, E91-D, 1963-1970.

26. Chai, Z.J.; Ouyang, Z.H.; Li, Z. Improved analysis of multi-objective particle swarm optimization based on Henon chaotic mapping. Ordnance Ind. Autom. 2020, 39, 48-52.

27. Feng, J.; Zhang, J.; Zhu, X.; Lian, W. A novel chaos optimization algorithm. Multimed. Tools Appl. 2017, 76, 17405-17436. [CrossRef]

28. Zhou, D.; Gao, X.; Liu, G.; Liu, Y. Randomization in particle swarm optimization for global search ability. Expert Syst. Appl. 2011, 38, 15356-15364. [CrossRef] 